\title{
Description, Implementation, and Evaluation of a Generic Design for Tabled CLP
}

\author{
Joaquín Arias ${ }^{1,2}$ \\ Manuel Carro ${ }^{1,2}$ \\ joaquin.arias@imdea.org, manuel.carro@\{imdea.org, upm.es\} \\ ${ }^{1}$ IMDEA Software Institute, ${ }^{2}$ Universidad Politécnica de Madrid
}

\begin{abstract}
Logic programming with tabling and constraints (TCLP, tabled constraint logic programming) has been shown to be more expressive and in some cases more efficient than LP, CLP or LP + tabling. Previous designs of TCLP systems did not fully use entailment to determine call / answer subsumption and did not provide a simple and well-documented interface to facilitate the integration of constraint solvers in existing tabling systems. We study the role of projection and entailment in the termination, soundness and completeness of TCLP systems, and present the design and an experimental evaluation of Mod TCLP, a framework that eases the integration of additional constraint solvers. Mod TCLP views constraint solvers as clients of the tabling system, which is generic w.r.t. the solver and only requires a clear interface from the latter. We validate our design by integrating four constraint solvers: a previously existing constraint solver for difference constraints, written in $\mathrm{C}$; the standard versions of Holzbaur's CLP(Q) and CLP(R), written in Prolog; and a new constraint solver for equations over finite lattices. We evaluate the performance of o ur framework in several benchmarks using the aforementioned constraint solvers. Mod TCLP is developed in Ciao Prolog, a robust, mature, next-generation Prolog system.
\end{abstract}

\section{Introduction}

Constraint Logic Programming (CLP) (Jaffar and Maher 1994) extends Logic Programming (LP) with variables which can belong to arbitrary constraint domains and whose associated constraint solvers can incrementally simplify equations set up during program executioCLP brings additional expressive power to LP, since constraints can very concisely capture complex relationships. Also, shifting from "generate-and-test" to "constrain-andgenerate" patterns reduces the search tree and therefore brings additional performance, even if constraint solving is in general more expensive than unification.

Tabling (Tamaki and Sato 1986; Warren 1992) is an execution strategy for logic programs which suspends repeated calls which could cause infinite loops. Answers from nonlooping branches are used to resume suspended calls which can, in turn, generate more answers. Only new answers are saved, and evaluation finishes when no new answers can 
be generated. Tabled evaluation always terminates for calls / programs with the bounded term depth property ${ }^{1}$ and can improve efficiency for terminating programs which repeat computations, as it automatically implements a variant of dynamic programming. Tabling has been successfully applied in a variety of contexts, including deductive databases, program analysis, semantic Web reasoning, and model checking (Warren et al. 1988; Dawson et al. 1996; Zou et al. 2005; Ramakrishna et al. 1997; Charatonik et al. 2002).

The combination of CLP and tabling (Toman 1997b; Schrijvers et al. 2008; Cui and Warren 2000; Chico de Guzmán et al. 2012) brings several advantages: it enhances termination properties, increases speed in a range of programs, and provides additional expressiveness. It has been applied in several areas, including constraint databases (Kanellakis et al. 1995; Toman 1997b), verification of timed automata and infinite systems (Charatonik et al. 2002), and abstract interpretation (Toman 1997a).

The theoretical basis of TCLP (Toman 1997b) was established in the framework of bottom-up evaluation of Datalog systems and presents the basic operations (projection and entailment checking) that are necessary to ensure completeness w.r.t. the declarative semantics. However, some previous implementations did not fully use these two operations (Schrijvers et al. 2008; Cui and Warren 2000), likely due to performance issues and also to the implementation difficulty.

On the other hand, previous TCLP frameworks featuring a more complete treatment of constraint projection and entailment (Chico de Guzmán et al. 2012) focused on adapting the implementation of a tabling algorithm to be used with constraints. As a result, and although the ideas therein were generic, they were not easily extensible. Adding new constraint domains to them is a difficult task that requires deep knowledge about the particular tabling implementation and the constraint solver. The modifications done to the tabling implementation for one particular constraint solver may very well be not useful for another constraint solver; in turn, constraint solvers had to be modified in order to make then aware of internal characteristics and capabilities of the tabling algorithm. These adaptations generate a technical debt that made using the full potential of TCLP very difficult.

In this work, we complete previous work on conditions for termination of TCLP, we provide a richer, more flexible answer management mechanism, we generalize the design of a tabling implementation so that it can use the projection and entailment operations provided by a constraint solver presented to the tabling engine as a server, and we define a set of operations that the constraint solver has to provide to the tabling engine. These operations are natural to the constraint solver, and when they are not already present, they should be easy to implement by extending the solver.

We have validatedour design (termed Mod TCLP) with an implementation in Ciao Pro$\log$ (Hermenegildo et al. 2012) where we interfaced four non-trivial constraint solvers to provide four different TCLP systems. We have experimentally evaluated these implementations with several benchmarks using TCLP. Graphical step by step executions of TCLP programs and performance comparison between TCLP interface are given in corresponding appendices, included in the supplementary material accompanying the paper at the TPLP archive.

\footnotetext{
${ }^{1}$ That is, programs which can only generate terms with a finite bound on their depth.
} 


\begin{tabular}{|l|c|c|c|c|c|}
\hline & LP & CLP & TAB & TCLP & Graph \\
\hline Left recursion & $\times$ & $\times$ & $\checkmark$ & $\checkmark$ & \multirow{2}{*}{ Without cycles } \\
\hline Right recursion & $\checkmark$ & $\checkmark$ & $\checkmark$ & $\checkmark$ & \\
\hline \hline Left recursion & $\times$ & $\times$ & $\times$ & $\checkmark$ & \multirow{2}{*}{ With cycles } \\
\hline Right recursion & $\times$ & $\checkmark$ & $\times$ & $\checkmark$ & \\
\hline
\end{tabular}

Table 1: Comparison of termination properties.

constraints; in turn, subsumption was shown to enhance termination and performance in tabling (Swift and Warren 2010). For example, the goal $G_{0} \equiv \operatorname{dist}(\mathrm{a}, \mathrm{Y}, \mathrm{D})$ is subsumed by $G_{1} \equiv \operatorname{dist}(\mathrm{X}, \mathrm{Y}, \mathrm{D})$ because the former is an instance of the latter $\left(G_{0} \sqsubseteq G_{1}\right)$. All the answers for $G_{1}$ where $\mathrm{X}=\mathrm{a}$ are valid answers for $G_{0}$; on the other hand, all the answers for $G_{0}$ are also answers for $G_{1}$.

The main idea behind the use of entailment in TCLP is that more particular calls (consumers) can suspend and later reuse the answers collected by more general calls (generators). In order to make this entailment relationship explicit, we define a TCLP goal as $\left\langle g, c_{g}\right\rangle$ where $g$ is the call (a literal) and $c_{g}$ is the projection of the current constraint store onto the variables of the call. Then, $\langle\operatorname{dist}(\mathrm{a}, \mathrm{Y}, \mathrm{D}), \mathrm{D}<150\rangle$ is entailed by the goal $\langle\operatorname{dist}(\mathrm{a}, \mathrm{Y}, \mathrm{D}), \mathrm{D}>0 \wedge \mathrm{D}<75\rangle$ because $\mathrm{D}>0 \wedge \mathrm{D}<75 \sqsubseteq \mathrm{D}<150$. We also say that the former (the generator) is more general than the latter (the consumer). All the solutions of the consumer are solutions of the generator or, in other words, the space of solutions of the consumer is a subset of that of the generator. However, not all the answers from a generator are valid for its consumers. For example $\mathrm{Y}=\mathrm{b} \wedge \mathrm{D}>125 \wedge \mathrm{D}<135$ is a solution for our generator, but not for our consumer, since the consumer call was made under a constraint store more restrictive than the generator. Therefore, the tabling engine should check and filter, via the constraint solver, that the answer from the generator is consistent w.r.t. the constraint store of the consumer.

The use of entailment in calls and answers enhances termination properties and can also increase speed (Section 6.1). The column "TCLP" in Table 1 summarizes the termination properties of dist/ 3 under TCLP, and shows that a full integration of tabling and CLP makes it possible to find all the solutions and finitely terminate in all the cases. Our TCLP framework not only facilitates the integration of constraint solvers with the tabling engine thanks to its simple interface (Section 4.1), but also minimizes the effort required to execute existing CLP programs under tabling (Section 5), since the changes required to the source code are minimal.

\section{Background}

In this section we present the syntax and semantics of constraint logic programs (Section 3.1), and extend the semantics and termination, soundness, and completeness proofs of (Toman 1997b) for a TCLP top-down execution (Sections 3.2 and 3.3, resp.). 


\subsection{Constraint Logic Programs}

Constraint logic programming (Jaffar and Maher 1994) introduces constraint solving methods in logic-based systems. A constraint logic program consists of clauses of the form:

$$
h:-c_{h}, l_{1}, \ldots, l_{k} \text {. }
$$

where $h$ is an atom, $c_{h}$ is a constraint, and $l_{i}$ are literals. The head of the clause is $h$ and the rest is called the body. The clauses where the body is always true, $h:-$ true, are called facts and usually written omitting the body $\left(h\right.$.). We will use $L$ to denote the set of $l_{i}$ in a clause. We will assume throughout this paper that the program has been rewritten so that clause heads are linearized (all the variables are different) and all head unifications take place in $c_{h}$. We will assume that we are dealing with definite programs, i.e., programs where the literals in the body are always positive (non-negated) atoms. Normal programsrequire a different treatment.

A query to a CLP program is a clause without head ?- $c_{q}, q_{1}, \ldots, q_{k}$, where $c_{q}$ is a constraint and $q_{i}$ are the literals in the query. We denote the set of $q_{i}$ as $Q$.

During the evaluation of a query to a CLP program, the inference engine generates constraints whose consistency with respect to the current constraint store is checked by the constraint solver. If the check fails, the engine backtracks to a previous state and takes a pending branch of the search tree.

A constraint solver, denoted by $\operatorname{CLP}(\mathcal{D})$, is a (partial) executable implementation of a constraint domain $\mathcal{D}$. A valuation $v$ over a set of variables $S=\left\{X_{1}, \ldots, X_{n}\right\}$ maps each variable $X_{i}$ to a value $d_{i}$ in $\mathcal{D}$, denoted by $v\left(X_{i}\right)=d_{i}$. $v$ is extended to expressions by substituting the variables in the expressions by the value they are mapped onto. A constraint can be a singleton constraint or a conjunction of simpler constraints. We denote constraints with lowercase letters and sets of constraints with uppercase letters. A solution of a constraint $c$ is a valuation $v$ over the variables occurring in $c$ if $v(c)$ holds in the constraint domain.

The minimal set of operations that we expect a constraint solver to support, in order to interface it successfully with our tabling system, are:

- Test for consistence or satisfiability: A constraint $c$ is consistent in the domain $\mathcal{D}$, denoted $\mathcal{D} \vDash c$, if $c$ has a solution in $\mathcal{D}$.

- Test for entailment $\left(\sqsubseteq_{\mathcal{D}}\right):^{3}$ We say that a constraint $c_{0}$ entails another constraint $c_{1}$ $\left(c_{0} \complement_{\mathcal{D}} c_{1}\right)$ if any solution of $c_{0}$ is also a solution of $c_{1}$. We extend the notion of constraint entailment to a set of constraints: a set of constraints $C_{0}$ entails another set of constraints $C_{1}$ (and we write it as $C_{0} \sqsubseteq_{\mathcal{D}} C_{1}$ ) if $\forall c_{i} \in C_{0} \exists c_{j} \in C_{1} . c_{i} \sqsubseteq_{\mathcal{D}} c_{j}$.

- An operation to compute the projection of a constraint $c$ onto a set of variables $S$ to obtain a constraint $c_{S}$ involving only variables in $S$ such that any solution of $c$ is also a solution of $c_{S}$, and a valuation $v$ over $S$ that is a solution of $c_{S}$ is a partial solution of $c$ (i.e., there exists an extension of $v$ that is a solution of $c$ ). We denote the projection as $\operatorname{Proj}(S, c)$.

\footnotetext{
${ }^{3}$ We may omit the subscript $\mathcal{D}$ if there is no ambiguity,
} 


\subsection{Semantics of CLP and TCLP}

The CLP fixpoint S-semantics (Falaschi et al. 1989; Toman 1997b) is defined as usual as the least fixpoint of the immediate consequence operators $S_{P}^{D}$ where all the operations behave as defined in the constraint domain $\mathcal{D}$ :

Definition 1 (Operator $S_{P}^{D}$ (Falaschi et al. 1989; Toman 1997b)). Let P be a CLP program and I an interpretation. The immediate consequence operator $S_{P}^{D}$ is defined as:

$$
\begin{aligned}
S_{P}^{\mathcal{D}}(I)=I \cup\{\langle h, c\rangle \mid & h:-c_{h}, l_{1}, \ldots, l_{k} \text { is a clause of } P, \\
& \left\langle a_{i}, c_{i}\right\rangle \in I, 0<i \leq k, \\
& c^{\prime}=\operatorname{Proj}\left(\operatorname{vars}(h), c_{h} \wedge \bigwedge_{i=1}^{k}\left(a_{i}=l_{i} \wedge c_{i}\right)\right), \\
& \mathcal{D} \vDash c^{\prime}, \\
& \text { if } \left.c^{\prime} \sqsubseteq c^{\prime \prime} \text { for some }\left\langle h, c^{\prime \prime}\right\rangle \in I \text { then } c=c^{\prime \prime} \text { else } c=c^{\prime}\right\}
\end{aligned}
$$

Note that $S_{P}^{\mathcal{D}}$ may not add a pair $\langle$ literal, const raint $\rangle$ when a more general constraint is already present in the interpretation being enlarged. However, to guarantee monotonicity, it does not remove existing, more particular constraints. The operational semantics of TCLP will however be able to do that.

The operational semantics of TCLP extends that of CLP programs under a top-down execution scheme (Jaffar and Maher 1994) that is defined in terms of a transition system between states:

Definition 2. A state is a tuple $\langle R, C\rangle$ where:

$-R$, the resolvent, is a multiset of literals and constraints that contains the collection of as-yet-unseen literals and constraints of the program. For brevity, when the set is a singleton we will write its only element using a lowercase letter instead of an uppercase letter, e.g. $t$ instead of $\{t\}$.

$-c$, the constraint store, is a (possibly empty) conjunction of constraints. It is acted upon by the constraint solver.

In (Jaffar and Maher 1994) the constraint store $c$ is divided in a collection of awake constraints and a collection of asleep constraints. This separation is ultimately motivated by implementation issues. We do not need to make that distinction here.

Given a query $\left\langle Q, c_{q}\right\rangle$, the initial state of the evaluation is $\left\langle Q, c_{q}\right\rangle$. Every transition step between states resolves literals of the resolvent against the clauses of the program and adds constraints to the constraint store. A derivation is successful if it is finite and the final state has the form $\langle\emptyset, c\rangle$ (i.e., the resolvent becomes empty). The answer to the query corresponding to this derivation is $c^{\prime}=\operatorname{Proj}(\operatorname{vars}(Q), c)$.

The transitions due to constraint handling are deterministic (there is only one possible descendant in every node), while the transitions due to literal matching are nondeterministic (there are as many descendants as clauses match with the node literal). We denote the set of tabled predicates in a TCLP program $P$ by $T a b_{P}$. The set of generators (calls to tabled predicates that do not entail previous calls), is denoted by GenP. The evaluation of a query to a TCLP program is usually represented as a forest of search trees, where each search tree corresponds to the evaluation of a generator and its nodes are the transitions states generated during the evaluation (see ?? of the supplementary material accompanying the paper at the TPLP archive, for some examples). 
The order in which the literals / constraints are selected is decided by the computation rule. During the computation of a TCLP program, two main phases are interleaved for the evaluation of every tabled goal: the call entailment phase (Def. 3) and the answer entailment phase (Def. 4).

Definition 3 (Call entailment phase). The call entailment phase checks if a new goal $\langle t, c\rangle$, where $t$ is a tabled literal (i.e., $t \in T a b_{P}$ ), entails a previous goal (called its generator). ${ }^{4}$ The new goal is resolved by answer resolution consuming the answers $c_{i}$ such that:

- $c_{i} \in A n s\left(g, c_{g}\right)$, where Ans $\left(g, c_{g}\right)$ is the set of answers of the oldest generator $\langle g, t\rangle \in G e n_{P}$, such that $g$ and $t$ are equal upon variable renaming, and $c \wedge(t=g) \sqsubseteq D c_{g}$, where $t=g$ is an abbreviation for the conjunction of equations between the corresponding arguments of t and $g$, i.e., $\left\langle g, c_{g}\right\rangle$ is more general than $\langle t, c\rangle$. In this case the goal $\langle t, c\rangle$ is marked as a consumer of $\left\langle g, c_{g}\right\rangle$.

- $\operatorname{Or} c_{i} \in A n s\left(t, c^{\prime}\right)$, where $c^{\prime}=\operatorname{Proj}(\operatorname{vars}(t), c)$ and Ans $\left(t, c^{\prime}\right)$ is the set of answers of a new generator $\left\langle t, c^{\prime}\right\rangle$ which is added to $G^{\prime} n_{P}$, the set of generator.

In TCLP, goals that match heads of tabled predicates are not resolved against program clauses. Instead, they are resolved consuming the answer constraints from a generator; this is termed answer resolution.

Definition 4 (Answer entailment phase). The answer constraints of a generator $\left\langle g, c_{g}\right\rangle$ are collected in the answer entailment phase in such a way that an answer which entails another more general answer is discarded / removed. The set of answers, denoted by Ans $\left(g, c_{g}\right)$, is the set of more general (w.r.t. $\left.\Xi_{D}\right)$ answer constraints $c_{i}^{\prime}$ obtained as the projection of $c_{i}$ onto vars $(g)$, where $\left\langle\emptyset, c_{i}\right\rangle$ (resp. $\left\langle\emptyset, c_{j}\right\rangle$ ) is the final state of a successful derivation:

$$
\begin{aligned}
\operatorname{Ans}\left(g, c_{g}\right)=\left\{c_{i}^{\prime} \mid\right. & c_{i}^{\prime}=\operatorname{Proj}\left(\operatorname{vars}(g), c_{i}\right), \\
& \left.\forall j \neq i, \nexists c_{j}^{\prime}=\operatorname{Proj}\left(\operatorname{vars}(g), c_{j}\right), c_{i}^{\prime} \sqsubseteq_{\mathcal{D}} c_{j}^{\prime}\right\}
\end{aligned}
$$

We will assume, without loss of generality, that the subscripts $i, j$ correspond to the order in which answers are found. The answer management strategy used in the answer entailment phase aims at keeping only the more general answers by discarding / removing more particular answers. This is specified by the quantification $\forall j \neq i$, where $i$ and $j$ are the indexes of the final constraint store. Simpler answer management strategies are possible: the implementations in (Cui and Warren 2000; Chico de Guzmán et al. 2012), following (Toman 1997b), only discard answers which are more particular than a previous one, i.e., they implement $\forall j<i$, and keep previously saved answers. A third possibility is to remove previous answers that are more particular than new ones, implementing $\forall j>i$. The choice among them does not impact soundness or completeness properties. However, discarding and removing redundant answers can greatly increase the efficiency of the implementation, as we experimentally show in section 6.3.

The order in which we search in the TCLP forest for a previous generator during the call entailment phase does not impact the completeness, soundness, or termination properties of the execution, but it can change its efficiency. Generators are naturally sorted from more

\footnotetext{
${ }^{4}$ Note that this entailment check includes subsumption in the Herbrand domain.
} 
particular ones (older) to more general (younger) ones - note that a younger, more particular call would be a consumer. Searching for a generator for a call can be performed in any direction. Starting at older, more particular generators, may need to examine several generators and perform potentially expensive entailment checks before finding one that suits the needs of the call. On the other hand, starting at younger, more general generators, should allow us to locate a suitable generator faster. However, this more general generator would have more answers associated which need to be filtered than what a more particular generator would have. Therefore, there does not seem to be a clear general strategy: either more generators have to be traversed, or more answers have to be filtered.

\subsection{Soundness, completeness and termination properties of TCLP}

(Toman 1997b) proves soundness and completeness of $S L G^{C}$ for TCLP Datalog programs by reduction to soundness and completeness of bottom-up evaluation. It is possible to extend these results to prove soundness and completeness of our proposal: they only differ in the answer management strategy and the construction of the TCLP forest. The strategy used in $S L G^{C}$ only discards answers which are more particular than a previous answer, while in our proposal we in addition remove previously existing more particular answers (Def. 4). The result of this is that only the most general answers are kept. In $S L G^{C}$, the generation of the forest is modeled as the application of rewriting rules.

Theorem 1 (Soundness w.r.t. the fixpoint semantics). Let $P$ be a TCLP definite program and $\left\langle q, c_{q}\right\rangle$ a query. Then for any answer $c^{\prime}$

$$
c^{\prime} \in \operatorname{Ans}\left(q, c_{q}\right) \Rightarrow \exists\langle q, c\rangle \in l f p\left(S_{P}^{\mathcal{D}}(\emptyset)\right) \cdot c^{\prime}=c_{q} \wedge c
$$

I.e., all the answers derived from the forest construction are also derived from the bottomup computation (and are therefore correct).

Answer resolution recovers constraints from the $\operatorname{Ans}\left(g, c_{g}\right)$ sets corresponding to a goal $g$ and an constraint $c_{g}$, instead of repeating SLD resolution on $\left\langle g, c_{g}\right\rangle$. These sets were ultimately generated by saving SLD resolution results, possibly using previously generated sets of answers for intermediate goals. ${ }^{5}$ Therefore, we can substitute any point where answers are recovered from $A n s\left(g, c_{g}\right)$ for the corresponding SLD resolution. So, if $c^{\prime} \in \operatorname{Ans}\left(q, c_{q}\right)$ then there is an SLD derivation $\left\langle q, c_{q}\right\rangle \rightsquigarrow\left\langle\emptyset, c^{\prime}\right\rangle$. Moreover, if $\langle q, \emptyset\rangle \rightsquigarrow\langle\emptyset, c\rangle$ then $c^{\prime}=c \wedge c_{q}$ and we can construct the answer to $\left\langle q, c_{q}\right\rangle$ from that of $\langle q, \emptyset\rangle$. So if $c^{\prime} \in \operatorname{Ans}\left(q, c_{q}\right)$, then there is $\langle q, \emptyset\rangle \rightsquigarrow\langle\emptyset, c\rangle$ and $c^{\prime}=c \wedge c_{q}$. From the correctness of SLD resolution, we have that if there is a derivation $\langle q, \emptyset\rangle \rightsquigarrow\langle\emptyset, c\rangle$ then $\langle q, c\rangle \in \operatorname{lfp}\left(S_{P}^{\mathcal{D}}(\emptyset)\right)$ and, as we said before, $c^{\prime}=c \wedge c_{q}$.

Theorem 2 (Completeness w.r.t. the fixpoint semantics). Let $P$ be a TCLP definite program and $\langle h$, true $\rangle$ a query. Then for every $\langle h, c\rangle$ in $l f p\left(S_{P}^{\mathcal{D}}\right)$ :

$$
\langle h, c\rangle \in l f p\left(S_{P}^{\mathcal{D}}(\emptyset)\right) \Rightarrow \exists c^{\prime} \in \text { Ans }(h, \text { true }) . c \sqsubseteq c^{\prime}
$$

I.e., all the answers derived from the bottom-up computation entail answers generated by the TCLP execution.

\footnotetext{
${ }^{5}$ Answers more particular than other answers may be removed, but those which remain are still correct answers.
} 
For any answer derived from the bottom-up computation $\langle h, c\rangle \in \operatorname{lf} p\left(S_{P}^{D}(\emptyset)\right)$ there exists a successful SLD derivation $\langle h$, true $\rangle \rightsquigarrow\langle\emptyset, c\rangle$. Since answer resolution may keep the most general answer when generating comparable answers (Def. 4), it is also complete if entailment with this most general answer is used instead of equality with the more particular answers (which were removed). Therefore, it will always be the case that $\exists c^{\prime} \in A n s(h$, true $) . c \sqsubseteq c^{\prime}$.

Termination of TCLP Datalog programs under a top-down strategy when the domain is constraint-compact (Def. 5) is proven in (Toman 1997b).

Definition 5 (Constraint-compact). Let $\mathcal{D}$ be a constraint domain, and $D$ the set of all constraints expressible in $\mathcal{D}$. Then $\mathcal{D}$ is constraint-compact iff:

- for every finite set of variables $S$, and

- for every subset $C \subseteq D$ such that $\forall c \in C$.vars $(c) \subseteq S$, there is a finite subset $C_{f i n} \subseteq C$ such that $\forall c \in C . \exists c^{\prime} \in C_{\text {fin }} . C \sqsubseteq D c^{\prime}$

In that case, the evaluation will suspend the exploration of a call whose constraint store is less general or comparable to a previous call. Eventually, the program will generate a set of call constraint stores which can cover any infinite set of constraints in the domain, therefore finishing evaluation. That is because, intuitively speaking, a domain $\mathcal{D}$ is constraint-compact if for any (potentially infinite) set of constraints $C$ expressible in $\mathcal{D}$, there is a finite set of constraints $C_{\text {fin }} \subseteq C$ that covers (in the sense of $\sqsubseteq_{\mathcal{D}}$ ) $C$. In other words, $C_{f i n}$ is as general as $C$.

Most TCLP applications require domains which are not constraint-compact because constraint-compact domains are not very expressive. Therefore, we refined the termination theorem (Theorem 23 in (Toman 1997b)) for Datalog programs with constraint-compact domains to cover cases where a program, during the evaluation, generates only a constraintcompact subset of all constraints expressible in the domain.

Theorem 3 (Termination). Let $P$ be a $T C L P(\mathcal{D})$ definite program and $\left\langle Q, c_{q}\right\rangle$ a query. Then the TCLP execution terminates iff:

- For every literal $g$, the set $C_{g}$ is constraint-compact, where $C_{g}$ is the set of all the constraint stores $c_{i}$, projected and renamed w.r.t. the arguments of $g$, s.t. $\left\langle g, c_{i}\right\rangle$ is in the forest $\mathcal{F}\left(Q, c_{q}\right)$.

- For every goal $\left\langle g, c_{g}\right\rangle$, the set $A_{\left\langle g, c_{g}\right\rangle}$ is constraint-compact, where $A_{\left\langle g, c_{g}\right\rangle}$ is the set of all the answer constraints $c^{\prime}$, projected and renamed w.r.t. the arguments of $g$, s.t. $c^{\prime}$ is a successful derivation in the forest $\mathcal{F}\left(Q, c_{q}\right)$.

The intuition is that for every subset $C$ of the set of all possible constraint stores $C_{g}$ that can be generated when evaluating a call to $P$, if there is a finite subset $C_{\text {fin }} \subseteq C$ that covers (i.e., is as general as) $C$, then, at some point, any call will entail previous calls, thereby allowing its suspension to avoid loops. Similarly, for every subset $A$ from the set of all possible answer constraints $A_{\left\langle g, c_{g}\right\rangle}$ that can be generated by a call, if there is a finite subset $A_{\text {fin }} \subseteq A$ that covers $A$, then, at some point, any answer will entail a previous one, ensuring 
entail the initial one after renaming since $(\mathrm{V}>-1 \wedge \mathrm{V}<9) \nsubseteq(\mathrm{X}>0 \wedge \mathrm{X}<10)$. Then this call is evaluated and produces the second recursive call, $\operatorname{nat}\left(\mathrm{Y}_{2}\right)$, $\left.\mathrm{X}>0 \wedge \mathrm{X}<10 \wedge \mathrm{X}=\mathrm{Y}_{1}+1 \wedge \mathrm{Y}_{1}=\mathrm{Y}_{2}+1\right\rangle$. Again, the projection of its constraint store, $\mathrm{Y}_{2}>-2 \wedge \mathrm{Y}_{2}<8$, does not entail any of the previous constraint stores, and so on. The evaluation therefore loops.

Example 3. The program in Fig. 3, left, also does not terminate for the query? - nat (X). Let us examine its behavior:

The answer constraint set is not compact The equation in the body of the clause $\mathrm{X}=\mathrm{Y}_{1}+1$ defines a relation between the variables but, since the domain of $\mathrm{X}$ is not restricted, its projection onto $\mathrm{Y}_{1}$ will return no constraints (i.e., Proj $\left(\mathrm{Y}_{1}, \mathrm{X}=\mathrm{Y}_{1}+1\right)=$ true). Therefore, the set of all call constraint stores generated by the query $\operatorname{nat}(\mathrm{X})$, true $\rangle$ is $C_{\text {nat }(\mathrm{v})}=\{$ true which is finite and constraint-compact. However, the answer constraint set $A_{\langle\text {nat }(\mathrm{v}), \text { true }\rangle}=\{\mathrm{V}=0, \mathrm{~V}=1, \ldots, \mathrm{V}=\mathrm{n}, \ldots\}$ is not constraint-compact.

Call suspension with an infinite answer constraint set The first recursive call is $\left\langle\operatorname{nat}\left(\mathrm{Y}_{1}\right), \mathrm{X}=\mathrm{Y}_{1}+1\right\rangle$ and the projection of its constraint store entails the initial store. Therefore, the TCLP evaluation suspends the recursive call, shifts execution to the second clause, and generates the answer $\mathrm{X}=0$. This answer is used to feed the suspended recursive call, resulting in the constraint store $\mathrm{X}=\mathrm{Y}_{1}+1 \wedge \mathrm{Y}_{1}=0$ which generates the answer $\mathrm{X}=1$. Each new answer $\mathrm{X}=\mathrm{n}$ is used to feed the suspended recursive call. Since the projection of the constraint stores on the call variables is true, the execution tries to generate infinitely many natural numbers. Therefore, the program does not terminate.

Example 4. Unlike the situation that happens in pure Prolog / variant tabling, adding new clauses to a program under TCLP can make it terminate. ${ }^{7}$ As an example, Fig. 3, right, is the same as Fig. 3, left, with the addition of the clause nat (X):- X \#> 1000. Let us examine its behavior under the query? - nat $(\mathrm{X})$ :

Compactness of call / answer constraint sets The set of all constraint stores generated remains $C_{\text {nat }(\mathrm{v})}=\{$ true $\}$. But the new clause makes the answer constraint set becomes $A_{\text {(nat(v), true }\rangle}=\{\mathrm{V}=0, \mathrm{~V}=1, \ldots, \mathrm{V}=\mathrm{n}, \ldots, \mathrm{V}>1000, \mathrm{~V}>1001, \ldots$, $\mathrm{V}>\mathrm{n}, \ldots\}$, which is constraint-compact because a constraint of the form $\mathrm{V}>\mathrm{n}$ is entailed by infinitely many constraints, i.e. it covers the infinite set $\{\mathrm{V}=\mathrm{n}+1, \ldots$, $\mathrm{V}>\mathrm{n}+1, \ldots\}$. Therefore, since both sets are constraint-compact, the program terminates.

First search, then consume The first recursive call $\left.\operatorname{nat}\left(\mathrm{Y}_{1}\right), \mathrm{X}=\mathrm{Y}_{1}+1\right\rangle$ is suspended and the TCLP evaluation shifts to the second clause which generates the answer $\mathrm{X}=0$. Then, instead of feeding the suspended call, the evaluation continues the search and shifts to the third clause, nat $(X):-X \# 1000$, and generates the answer $\mathrm{X}>1000$. Since no more clauses remain to be explored, the answer $\mathrm{X}=0$

\footnotetext{
${ }^{7}$ This depends on the strategy used by the TCLP engine to resume suspended goals. Our implementation gathers all the answers for goals that can produce results first, and then these answers are used to feed suspended goals. This makes the exploration of the forests proceed in a breadth-first fashion.
} 
store_projection(+Vars, -ProjStore) Returns in ProjStore a representation of the projection of the current constraint store onto the list of variables Vars.

call_entail (+ProjStore, +ProjStore gen $_{\text {) }}$ Succeeds if the projection of the current constraint store, ProjStore, entails the projected store, ProjStore ${ }_{\text {gen }}$, of a previous generator. It fails otherwise.

answer_compare (+ProjStore, +ProjStore ans, -Res) Returns Res='s $=<$ if the projected store of the current answer, ProjStore, entails the projected store of a previous answer, ProjStore $e_{a n s}$, or Res='>' if ProjStore is entailed by ProjStore ans and they are not equal. It fails otherwise.

apply_answer(+Vars, +ProjStore) Adds the projected constraint store ProjStore of the answer to the current constraint store and succeeds if the resulting constraint store is consistent.

Fig. 4: Generic interface specification.

is used, generating $\mathrm{X}=1$. Then $\mathrm{X}>1000$ is used, resulting in the constraint store $\mathrm{X}=\mathrm{Y}_{1}+1 \wedge \mathrm{Y}_{1}>1000$, which generates the answer $\mathrm{X}>1001$. However, during the answer entailment phase, $\mathrm{X}>1001$ is discarded because $\mathrm{X}>1001 \sqsubseteq \mathrm{X}>1000$. Then, one by one each answer $\mathrm{X}=\mathrm{n}$ is used, generating $\mathrm{X}=\mathrm{n}+1$. But when the answer $\mathrm{X}=1000$ is used, the resulting answer $\mathrm{X}=1001$ is discarded, during the answer entailment phase, because $\mathrm{X}=1001 \sqsubseteq \mathrm{X}>1000$. At this point the evaluation terminates because there are no more answers to be consumed. The resulting set of answers is Ans(nat $(\mathrm{X})$, true $)=\{\mathrm{X}=0, \mathrm{X}>1000, \mathrm{X}=1, \ldots, \mathrm{X}=1000\}$

\section{The Mod TCLP Framework}

In this section we describe the Mod TCLP framework, the operations required by the interface, and the program transformation that we use to compile programs with tabled constraints (Section 4.1). We also provide a sketch of its implementation and we describe step-by-step some executions at the level of the TCLP libraries (Section 4.2 and ?? of the supplementary material). In Section 4.3 we present the implementation of the TCLP interface for Holzbaur's CLP(Q) solver and in Section 4.4 we present an optimization, the Two-Step projection.

\subsection{Design of the Generic Interface}

Mod TCLP provides a generic interface (Fig. 4) designed to facilitate the integration of different constraint solvers. The predicates of the interface use extensively two objects: Vars, the list of constrained variables, provided by the tabling engine to the constraint solver, and ProjStore, a representation of the projected constraint store, opaque to the tabling engine, and which should be self-contained and independent (e.g., with fresh variables) from the main constraint store. For example, the constraint solver CLP(D $\left.D_{\leq}\right)$(Section 5.1) is written in $\mathrm{C}$ and the projection of a constraint store is a $\mathrm{C}$ structure whose representation is its memory address and length. 
To implement these predicates, the constraint solver has to support the (minimal) set of operations defined in Section 3.1: projection, test for entailment, and test for consistence. The predicates that the constraint solver must provide in order to enable its interaction with the tabling engine are:

- store_projection(+Vars, -ProjStore), that is invoked before the call and the answer entailment phases:

- It is used before the call entailment phase to generate the representation of the goal as a tuple $\langle$ G, ProjStore $\rangle$, where ProjStore represents the projection of the constraint store at the moment of the call onto Vars, the variables in G. Although a generic implementation should include the Herbrand constraints of the call in the constraint store, our implementation does not consider Herbrand constraints to be part of the constraint store by default. Instead, calls are syntactically compared using variant checking, but the programmer can also choose to use subsumption, if required, by using a package described below. There are some reasons for that decision: on the one hand, programmers (even using tabling) are used to this behavior; on the other hand, there are data structures highly optimized (Ramakrishnan et al. 1995) to save and retrieve calls together with their input / output substitutions which perform variant checking on the fly while taking advantage of the WAM-level representation of substitutions.

- Similarly, before the answer entailment phase, the projection of the Herbrand constraints onto the variables of the goal is directly taken care of by their WAM-level representation. We use variant checking to detect when the Herbrand constraints associated to two calls are equal. Therefore, an answer constraint is internally represented by a tuple $\langle\mathrm{S}$, ProjStore $\rangle$ where $\mathrm{S}$ captures the Herbrand constraints of the variables of the goal and ProjStore represents the projection of the rest of the answer constraint onto Vars, the variables of the answer.

- call_entail (+ProjStore, +ProjStore gen $_{\text {) }}$ ) is invoked during the call entailment phase to check if a new call, represented by $\langle G$, ProjStore $\rangle$, entails a previous generator, represented by $\left\langle\mathrm{G}_{\mathrm{gen}}\right.$, ProjStore $\left.{ }_{\text {gen }}\right\rangle$, where $\mathrm{G}$ is a variant of $\mathrm{G}_{\mathrm{gen}}$. The

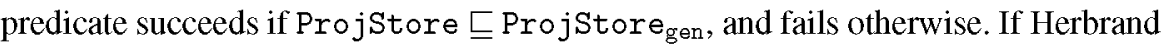
subsumption checking is needed, our implementation provides a package which transforms calls to tabled predicates so that suspension is based on entailment in IH. This transformation moves Herbrand constraint handling away from the level of the WAM by creating attributed variables (Cui and Warren 2000) that carry the constraints - i.e., the unifications. Later on, a Herbrand constraint solver is used to check subsumption. ${ }^{8}$

- answer_compare (+ProjStore, +ProjStore ans, -Res) is invoked during the answer entailment phase to check a new answer, represented by $\langle\mathrm{S}$,

\footnotetext{
${ }^{8}$ If there are several constraint domains involved, such as e.g. CLP(H) and CLP(Q), we assume that we can distinguish them appropriately at run-time and the entailment is determined as variant $\left(\mathrm{G}, \mathrm{G}_{\text {gen }}\right) \wedge$ ProjStore $\sqsubseteq_{H}$ ProjStore $_{\text {gen }} \wedge$ ProjStore $\sqsubseteq_{\mathrm{Q}}$ ProjStore $_{\text {gen }}$
} 
ProjStore $\rangle$, against a previous one, represented by $\left\langle S_{a n s}\right.$, ProjStore $\left.{ }_{a n s}\right\rangle$, when the Herbrand constraints $S$ and $S_{a n s}$ are equal. The predicate compares ProjStore and ProjStoreans and returns ' $=<$ ' in its last argument when ProjStore $\sqsubseteq$ ProjStore $a n s$, ' $>$ ' when ProjStore $\sqsupset$ ProjStore $_{\text {ans }}$, and fails otherwise. This bidirectional entailment check, which is used to discard / remove more particular answers, is a potentially costly process, but it brings considerable advantage from saved resumptions (Section 6.3): when an answer is added to a generator, consumers are resumed by that answer. These consumers in turn generate more answers and cause further resumptions in cascade. Reducing the number of redundant answers reduces the number of redundant resumptions, and we have experimentally observed that it brings about important savings in execution time.

- apply_answer (+Vars, +ProjStore) is invoked to consume an answer from a generator. In variant tabling, since consumers are variants of generators, answer substitutions from generators can always be applied to consumers. That is not the case when using entailment in TCLP: consumers may be called in the realm of a constraint store more restrictive than that of their generators, and answers from the generator have to be filtered to discard those which are inconsistent with the constraint store at the time of the call to the consumer. In our implementation, an answer is represented by $\langle\mathrm{S}$, ProjStore $\rangle$, where $\mathrm{S}$, the set of Herbrand constraints, is applied by the tabling engine and ProjStore, the projection of the constraint answer, is added to the constraint store of the consumer by apply_answer $/ 2$, which succeeds iff the resulting constraint store is consistent.

The design of the interface assumes that external constraint solvers are compatible with Prolog operational semantics so that when Prolog backtracks to a previous state, the corresponding constraint store is transparently restored. That can be done by adding a Prolog layer which uses the trail to store undo information which is used to reconstruct the previous constraint store when Prolog backtracks (this is a reasonable, minimal assumption for any integration of constraint solving and logic programming). The TCLP interface then can readily use any constraint solver which follows this design, because the suspension and resumption mechanisms of the tabling are based on the trailing mechanism of Prolog. When a consumer suspends, backtracking takes place, the memory stacks are frozen, and the variable bindings are saved on untrailing. They are reinstalled upon consumer resumption.

However, the entailment operations call_entail/2 and answer_compare/3 need to know the correspondence among variables in ProjStore and in ProjStore gen (resp., ProjStore $_{a n s}$ ). To this end, projections are (conceptually) a pair (VarList, Store), where VarList is a list of fresh variables in Store that correspond to Vars, the variables on which the projection was originally made. Different, independent constraint stores can then be compared by means of these lists. This list is also necessary to apply the ProjStore of an answer to the global store: it is used to determine the correspondence of variables between the global and the projected store.

The actual implementation may differ among constraint solvers. For example, the TCLP(Q) interface (Fig. 9) uses a list of fresh variables following the same order as those 
in Vars. However, the TCLP( $\left(\mathbb{D}_{\leq}\right)$interface (Section 5.1) uses a vector containing the index in the matrix corresponding to every variable in Vars, again following the same order.

\subsection{Implementation Sketch}

We summarily describe now the implementation of Mod TCLP, including the global table where generators, consumers, and answers are saved. We also present the transformation performed to execute tabled predicates and a (simplified) flowchart showing the interactions between the tabling engine and the constraint solver through the generic interface.

\subsubsection{Global Table}

Tries are the data structure of choice for the call / answer global table (Ramakrishnan et al. 1995). In variant tabling, every generator $G_{g e n}$ is uniquely associated (modulo variable renaming) to a leaf from where the Herbrand constraints for every answer hangs. Generators are identified in Mod TCLP by the projection of the constraint store on the variables of the generator, i.e., with a tuple $\left\langle G_{g e n}\right.$, ProjStore $\left.e_{\text {gen }}\right\rangle$. We store generators in a trie where each leaf is associated to a call pattern $G_{g e n}$ and a list with a frame for each projected constraint store ProjStore $\operatorname{gen}_{i}$. Each frame identifies: (i) the projected constraint store ProjStore gen $_{i}$, (ii) the answer table where the generator's answers Ans $\left(\mathrm{G}_{\mathrm{gen}}\right.$, ProjStore gen $\left._{\mathrm{i}}\right)$ are stored, and (iii) the list of its consumers.

Answers are represented by a tuple $\left\langle\mathrm{S}_{\text {ans }}\right.$, ProjStore $\left.{ }_{a n s}\right\rangle$ and stored in a trie where each leaf points to the Herbrand constraints $S_{a n s}$ and to a list with the projected constraint stores ProjStore ${ }_{\text {ans }_{i}}$ corresponding to answers whose Herbrand constraints are a variant of $S_{\text {ans }}$. The answers are stored in order of generation, since (as we will see later) it is not clear that other orders eventually pay off in terms of speeding up the entailment check of future answers.

\subsubsection{TCLP Directives and Program Transformation}

Executing a CLP program under the TCLP framework only needs to enable tabling and import a package which implements the bridge CLP / tabling instead of the regular constraint solver. Fig. 5, left, shows the TCLP version of the left recursive distance traversal program in Fig. 1, right. The constraint interface remains unchanged, and the program code does not need to be modified to be executed under TCLP. The directive :- use_package(tabling) initializes the tabling engine and the directive :- use_package ( $\left.t_{-} c l p q\right)$ imports TCLP(Q), the TCLP interface for the CLP(Q) solver (Section 4.3). To select another TCLP interface (more examples in Section 5) we just have to import the corresponding package. Finally, the directive :- table dist/3. specifies that the predicate dist $/ 3$ should be tabled.

Fig. 5, right, shows the transformation applied to the predicate dist/3. The original entry point to the predicate is rewritten to call an auxiliary predicate through the metapredicate tabled_call/1 (Fig. 6). The auxiliary predicate corresponds to the original one with a renamed head and with an additional new_answer/0 (Fig. 7) at the end of the 


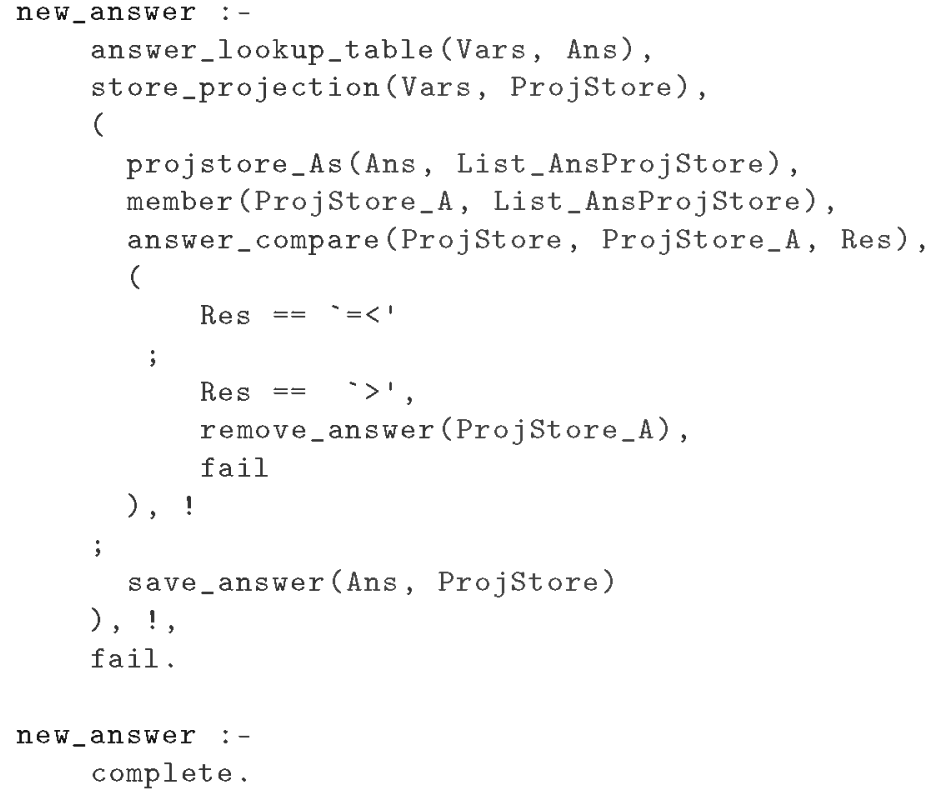

Fig. 7: Implementation of new_answer/0.

2. The tabling engine calls store_projection/2, which returns in ProjStore the projection onto Vars of the current constraint store.

3. The tabling engine uses member/2 to retrieve in ProjStore_G the projected constraint stores from the list of frames associated to Gen. If it succeeds, the execution continues in step 5. If it fails, it may be because Gen is the first occurrence of this call pattern, or because it does not entail any of the previous generators (and it is therefore a new generator).

4. The tabling engine calls save_generator $/ 3$ to add a new frame to Gen, identifying the new call as a generator. The projected store ProjStore is saved in this new frame and the answer table and the consumer list are initialized. From this point on, the generator is identified by $\left\langle\right.$ Gen, ProjStore P $_{G}$ ) and the execution continues in step 7.

5. The constraint solver checks if the current store ProjStore entails the retrieved projected constraint store ProjStore_G using call_entail/2. In that case, Call is suspended in step 6 . Otherwise, the tabling engine tries to retrieve another projected constraint store in step 3.

6. If the generator is not complete, the tabling engine suspends the execution of Call with suspend_consumer/ 1 and adds Call to the list of consumers of the generator. Execution then continues by backtracking over the youngest generator. Otherwise, Call continues the execution in step 14. A suspended consumer is resumed when its generator produces new answers, and also continues in step 14.

7. The generator $\langle$ Gen, ProjStore_G $\rangle$ is executed with execute_generator $/ 2$, which calls the renamed tabled predicate, and its reference is pushed onto the PTCP stack. 


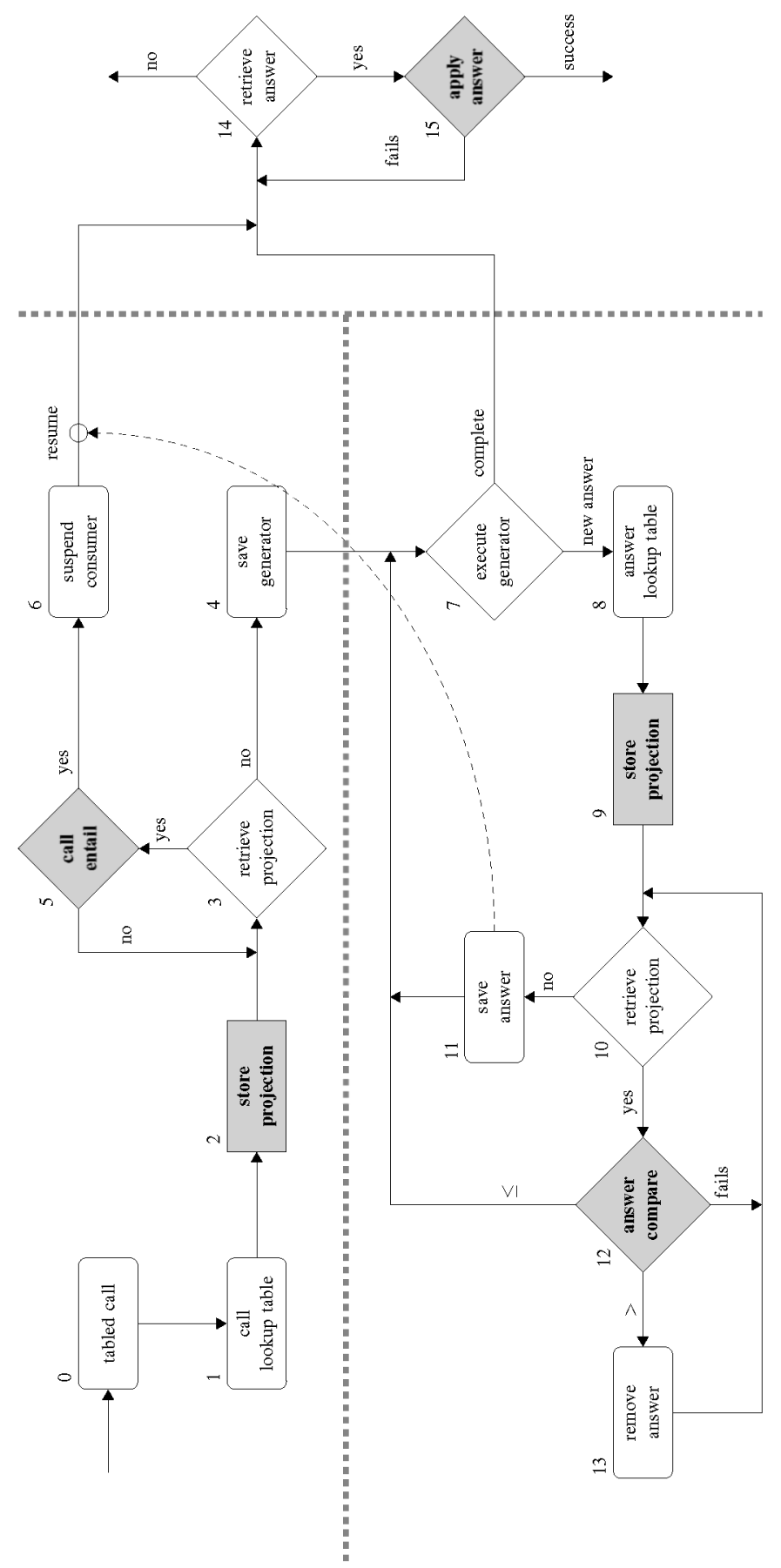

Fig. 8: Flowchart of the execution algorithm of Mod TCLP. 
If the execution reaches the end of a clause, a new answer has been found and new_answer $/ 0$ continues the execution in step 8.

8. This is the entry point for new_answer/0. The tabling engine calls answer_lookup_table/2, which retrieves a reference to the generator in execution from the PTCP stack. A reference to the Herbrand constraints of the current answer in the generator's answer table is returned in Ans, and the list of variables from the call that are now/still constrained is returned in Vars.

9. The tabling engine invokes store_projection/2. This returns in ProjStore the projection of the current constraint store onto the constrained variables of the answer, Vars.

10. The tabling engine retrieves from Ans the list of projected constraint stores in List_AnsProjStore and calls member $/ 2$ to return the stores one at a time in ProjStore_A. If it succeeds, the execution continues in step 12; otherwise, it continues in step 11. Failure can happen because all projected constraint stores were already retrieved from List_AnsProjStore or because Ans is the first answer with these Herbrand constraints.

11. The tabling engine adds ProjStore to the list of projected constraint stores (List_AnsProjStore) of the corresponding Ans with save_answer/2, and resumes one by one the consumers of the current generator which were suspended in step 6 . Since new_answer $/ 0$ always fails, the execution backtracks to complete the execution of the generator (step 7).

12. The constraint solver checks if the current store ProjStore entails the retrieved projected constraint store ProjStore_A using answer_compare/3. If this is the case, it returns Res $=$ ' $=<$ ', which makes new_answer/0 discard the current answer, and the generator is re-executed in step 7. If ProjStore is entailed by ProjStore_A and they are not equal, it returns Res = ' $>$ ' and ProjStore_A is removed in step 13. Otherwise, it fails and the execution continues in step 10, where the tabling engine tries to retrieve another projected constraint store.

13. The tabling engine marks the more particular answer as removed using remove_answer/1. Then the execution continues in step 10.

14. Once the generator has exhausted all the answers and does not have more dependencies, it is marked as complete using complete/0 and the generator's reference is popped from the PTCP stack. The tabling engine retrieves answers $\langle$ Ans, ProjStore_A . from the generator 〈Gen, ProjStore_G〉 using member $/ 2$. If it succeeds and the answer is not marked as removed, the answer will be applied in step 15. Otherwise, the execution backtracks to retrieve another answer.

15. Applying the Herbrand constraints Ans always succeeds, because the generator and its consumers have the same call pattern. Then the constraint solver adds the projected constraint store of the answer to the current constraint store with apply_answer $/ 2$, and checks if the resulting constraint store is consistent. If so, execution continues; otherwise the execution goes back to step 14 .

In the ?? of the supplementary material accompanying the paper at the TPLP archive, we will walk through a step by step execution of a program using the TCLP(Q) interface described in Section 4.3. 


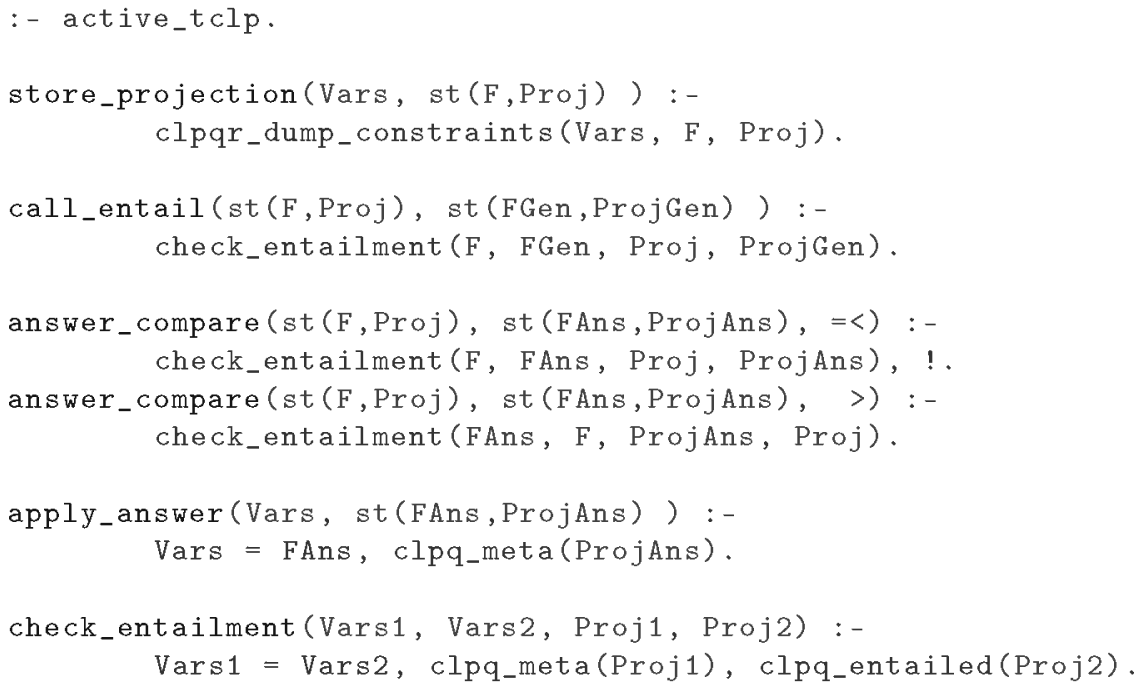

Fig. 9: The Mod TCLP interface for CLP(Q) is a bridge to existing predicates.

\subsection{Implementation of the TCLP $(\mathbb{Q})$ Interface}

Fig. 9 shows the interface for Holzbaur's CLP(Q) solver (Holzbaur 1995) as an example of integration of a constraint solver with Mod TCLP. This CLP(Q) implementation already provides most of the functionality required by the tabling engine, and therefore the TCLP(Q) interface actually acts as a bridge to existing predicates.

A Mod TCLP constraint interface starts with the declaration :- active_tclp. It makes the compiler check which interface predicates are available in order to adjust the program transformation, and instructs the run-time to activate the TCLP framework. The functionality required by the interface is implemented as follows:

- store_projection(+Vars,-st(F,Proj)) calls the CLP(Q) predicate clpqr_dump_constraints (+Vars, $-F,-$ Proj) to perform the projection. It returns in Proj the projection of the current store onto the list of variables Vars. The variables in Proj are fresh and are contained in the list F, which follows the same order as those in Vars and can be used to restore the association between the variables in Vars and the constraints in Proj, as we said in Section 4.1.

- call_entail(+st(F,Proj),+st(FGen,ProjGen)) calls the auxiliary predicate check_entailment (F, FGen, Proj, ProjGen) which success if Proj $\sqsubseteq$ ProjGen. First, check_entailment/4 unifies F and FGen, resp. the variables of the projection of the current store, and the variables of the generator's projection. Then, the CLP(Q) predicate clpq_meta (+Proj) makes Proj part of the current constraint store by executing it. This does not interact with the current store, because the variables in F and FGen are fresh. And finally, clpq_entailed (+ProjGen) success if ProjGen is entailed by the current constraint store (i.e., Proj $\sqsubseteq$ ProjGen).

- answer_compare (+st (F,Proj), +st (FAns, ProjAns), Res) calls the predicate check_entailment(F, FAns, Proj, ProjAns) to check if Proj $\sqsubseteq$ ProjAns. 
If it is the case, answer_compare/3 returns $=<$ in Res. Otherwise, it calls check_entailment (FAns, F, ProjAns, Proj) to check if ProjAns $\sqsubset$ Proj. If it is the case, it returns > in Res, otherwise it fails (i.e., there is no entailment in any direction).

- apply_answer(+Vars, +st(FAns, ProjAns)) unifies FAns, the variables of ProjAns with Vars, those in the pattern of the resumed call. Then, it uses the CLP(Q) predicate clpq_meta(+ProjAns) to add the answer constraint store ProjAns to the current constraint store. If the resulting constraint store is consistent, execution continues, and it fails otherwise.

The TCLP interface for CLP(R) is similar to that of CLP(Q). CLP(R) uses floatingpoint numbers and its performance is better than that of CLP(Q), which uses exact fractions. However, floating-point rounding errors make CLP(R) (and TCLP(R)) inappropriate for some applications, as entailment is unsound and therefore termination can be compromised.

\subsection{Two-Step Projection}

The design we have presented strives for simplicity. There is however an improvement that can be used to obtain more performance / reduce memory usage, at the cost of a slightly more complex design. We present it now, with the understanding that it does not change the general ideas we have presented so far.

store_projection/2 is usually the most expensive operation in the TCLP interface, but it is only mandatory when a call is a generator, which we can determine from entailment checking. ${ }^{9}$ We have however placed store_projection/ 2 before entailment checking because constraint solvers can often use the projection operation to compute some information needed by the entailment check. Instead of recomputing this information, the projection is divided in two parts: an initial operation early_call_projection(+Vars, -EarlyProj), executed before the entailment phase, that returns in EarlyProj the information needed to check entailment, and a second operation final_call_projection(+Vars, +EarlyProj, -ProjStore) that is executed after the entailment phase if the entailment check fails (and the call would then be a generator). If it is executed, this operation returns the projected constraint store in ProjStore using the information in EarlyProj.

For symmetry, a similar mechanism is used with answers. Instead of using store_projection/2 (step 10 in Fig. 8), two specialized versions are expected used: early_ans_projection/2, is called before the answer entailment check and final_ans_projection/3, called after the answer entailment check.

Example 5. The TCLP $(\mathrm{Q})$ interface in Fig. 9 uses store_projection/2 to project the constraint store of every new call. But, since clpq_entailed/1 does not need the projection of the current constraint store to check entailment w.r.t. the projected constraint store of a previous generator, the execution of the projection can be delayed:

\footnotetext{
${ }^{9}$ For efficiency, we can check entailment using the current constraint store $A$ instead of its projection onto a set of variables $S$ because $A \sqsubseteq B \Longleftrightarrow \operatorname{Proj}(S, A) \sqsubseteq B$, where $S=\operatorname{var}(B)$, as in our case,
} 


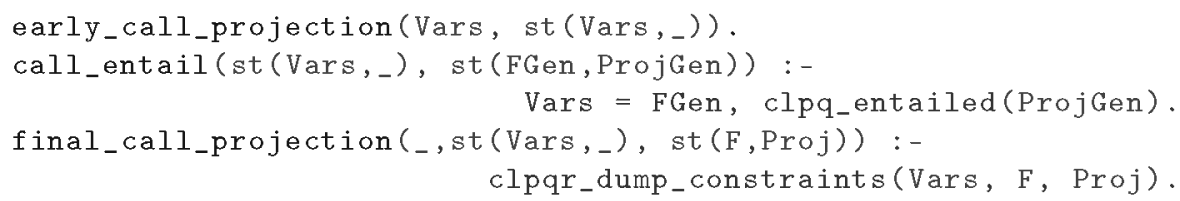

The performance impact of implementing the Two-Step projection is evaluated in Section 6.4, using the TCLP(Q) interface.

\section{Other TCLP Interfaces}

The design we presented brings more flexibility to a system with tabled constraints at a reasonable cost in implementation effort. To support this claim we present the implementation of the TCLP interface for a couple of additional solvers: a constraint solver for difference constraints (Section 5.1) completely written in C and ported from (Chico de Guzmán et al. 2012), and a solver for constraints over finite lattices (Section 5.2).

\subsection{Difference Constraints}

Difference constraints $\operatorname{CLP}\left(\mathbb{D}_{\leq}\right)$is a simple but relatively powerful constraint system whose constraints are generated from the set $\mathcal{C}_{\mathbb{D}_{<}}=\{X-Y \leq d: X, Y, d \in \mathbb{Z}\}$, where $X$ and $Y$ are variables, and $d$ is a constant.

A system of difference constraints can be modeled with a weighted graph, and it is satisfiable if there are no cycles with negative weight. A solver for this constraint system can be based on shortest-path algorithms (Frigioni et al. 1998) where the constraint store is represented as an $n \times n$ matrix A of distances. The projection of a constraint store A onto a set of variables $\mathrm{V}$ extracts a sub-matrix A' containing all pairs $\left(v_{1}, v_{2}\right)$ s.t. $v_{1}, v_{2} \in V$. For efficiency, a projection can be represented as a vector of length $|V|$ containing the index of each $v_{i}$ in $\mathrm{A}$. For example, if the indexes in $\mathrm{A}$ of the variables $[\mathrm{X}, \mathrm{Y}, \mathrm{Z}, \mathrm{T}, \mathrm{W}]$ are $(1,2,3,4,5)$, the projection onto the set of variables $[T, X, Y]$ is represented with the vector $(4,1,2)$. The implementation uses attributed variables to map Prolog variables onto their representation in the matrix by having as attribute the index of each variable in the matrix. Therefore, calculating projection is fast.

The TCLP( $\left(\mathbb{D}_{\leq}\right)$interface showcases that, as we mentioned in Section 4.1, the representation of the projected constraint store depends on the constraint solver. In this case, the projected constraint store is represented by a triple st ( $I d, L n, \operatorname{Proj}$ ) where Id is the memory address of the vector with the indexes of the constrained variables of the call / answer, Ln is its length (the number of constrained variables), and Proj is the memory address of a copy of the sub-matrix which represents the projected constraint store. The indexes of the vector Id follow the same order as the variables in Vars and are used to restore the association between Vars and Proj when they have to be compared or applied.

$\operatorname{CLP}\left(\mathbb{D}_{\leq}\right)$checks entailment using clpdiff_entailed((Id,Ln),ProjGen) and clpqdiff_entails ( $(I d, L n), P r o j G e n)$, where Id and Ln identify the position of the variables in the matrix A (the current constraint store), and ProjGen is the memory address of a sub-matrix which represent the projection of a previous generator. Note that the 
indexes of the sub-matrix from 1 to $n$ follows the order of the indexes in Id, i.e., the $k$ column /row of the sub-matrix correspond to the variable identified by the $k$ index in Id.

Therefore, the TCLP( $\left.\mathbb{D}_{\leq}\right)$interface increases performance and reduces memory footprint using the Two-Step projection (Section 4.4) because it only makes a copy of the sub-matrix Proj when the entailment phase fails and the current call / answer becomes a generator / new answer. See below the implementation of the projection and answer comparison operations using the Two-Step projection in the answer entailment check:

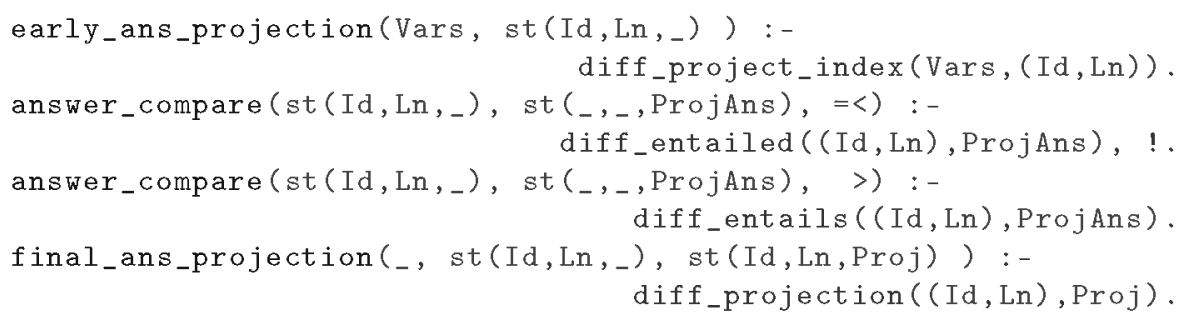

\subsection{Constraints over Finite Lattices}

A lattice is a triple $(\mathbb{S}, \sqcup, \sqcap)$ where $\mathbb{S}$ is a set of points and join $(\sqcup)$ and meet $(\sqcap)$ are two internal operations that follow the commutative, associative and absorption laws. ( $\mathbb{S}$, $\sqsubseteq$ ) is a poset where $\forall a, b \in \mathbb{S} . a \sqsubseteq b$ if $a=a \sqcap b$ or $b=a \sqcup b$ and $\exists \perp, \top \in \mathbb{S}$ such that $\forall a \in \mathbb{S} . \perp \sqsubseteq a \sqsubseteq \top$.

In the system of constraints over finite lattices CLP ( $\mathbb{L} a t$ ), the constraints between points in the lattice arise from (1) the topological relationship of the lattice elements and (2) any additional operations between the elements in the lattice. These two classes of constraints are handled by two different layers.

The external layer is concerned with the lattice topology and implements the constraint $Y \sqsubseteq X$ with $X, Y \in \mathbb{S}$ and the projection operation for variable elimination using Fourier's algorithm (Marriott and Stuckey 1998): the projection of $X \sqsubseteq d \wedge Y \sqsubseteq X$ onto $Y$ is $Y \sqsubseteq d$. This layer provides entailment checking and the operation to add a projected constraint store to the current constraint store.

Further constraints on variables can be imposed by relationships derived from internal operations other than those in the lattice. Compare, for example, $Y \sqsubseteq X$ with $Y \sqsubseteq X \wedge$ $Y=X \oplus X$ for some operation $\oplus$ among elements of the lattice: the additional information can be helpful to simplify (or prove inconsistent) the constraint store. In the lattice solver, a second layer implements these additional operations (if they exist) and communicates with the topology-related layer.

We have used this solver to implement a constraint tabling-based abstract interpreter (Section 6.5), where the points of the lattice are the elements of the abstract domain. The lattice implementation provides at least the operators $\sqcup$ and $\sqcap$ and the operations among the elements of the lattice, which are the counterparts of the operations in the concrete domain, as described above. 


\section{Experimental Evaluation}

In this section we evaluate the performance of our framework using the four constraint systems and interfaces we have summarily described $\left(\mathbb{Q}, \mathbb{R}, \mathbb{D}_{\leq}\right.$and $\left.\mathbb{L} a t\right)$.

In Section 6.1, we quantify the performance benefits of TCLP versus LP, tabling, and CLP using the dist/3 (Fig. 1) program presented in section 2 with the TCLP(Q) interface. Then we explore the impact and advantages of a more flexible modular framework. On the one hand, in Section 6.2 we evaluate the performance impact of the increased overhead w.r.t. previous implementations with less flexibility (i.e., the previous TCLP implementation of (Chico de Guzmán et al. 2012)) and, on the other hand, in Section 6.3 we evaluate the benefits of a more complete answer management strategy.

In Section 6.4, we evaluate the performance benefits of the Two Step projection using TCLP(Q). These benefits are due to the reduction in the number of projections executed during the evaluation. In Section 6.5, we use tabling and the new TCLP(Lat $)$ interface to implement a simple abstract interpreter, which we benchmark.

In ?? of the supplementary material accompanying the paper at the TPLP archive, we compare the expressiveness and performance of the TCLP( $\left.\mathbb{D}_{\leq}\right)$, TCLP $(R)$, and TCLP(Q) interfaces. In this case, the expressiveness of CLP(R/Q) comes with an overhead (which is higher in $\operatorname{CLP}(\mathbb{Q})$ due to its higher precision), but in certain problems this expressiveness can bring greats benefits using TCLP (additionally, in some problems the precision could be determinant).

The Mod TCLP framework presented in this paper is implemented in Ciao Prolog. The benchmarks and a Ciao Prolog distribution including the libraries and interfaces presented in this paper are available at http://www.cliplab.org/papers/tplp2018-tclp/. ${ }^{10} \mathrm{All}$ the experiments were performed on a Mac OS-X 10.9.5 machine with a $2.66 \mathrm{GHz}$ Intel Core 2 Duo processor. Times are given in milliseconds.

\subsection{Absolute Performance of TCLP vs. LP vs. Tabling vs. CLP}

Let us recall Table 1, where we used the dist/3 program (Fig. 1) to support the use of TCLP due to its better termination behavior. We now want to check whether, for those cases where LP or CLP also terminate, the performance of TCLP is competitive and for those cases where only TCLP terminates, whether its performance is reasonable. We have used a graph of 35 nodes without cycles (775 edges) and a graph of 49 nodes with cycles (785 edges) and timed the results - see Table 2.

As we already saw in Table 1, TCLP not only terminates in all cases, but it is also faster than the rest of the frameworks due to the combination of tabling (which avoids entering loops and caches intermediate results) and constraint solving. It also suggests, in line with the experience in tabling, that left-recursive implementations are usually faster and preferable, as they avoid work by "suspending first" and reusing answers when they are ready.

${ }^{10}$ Stable versions of Ciao Prolog are available at http://www . ciao- lang . org. However, The libraries and interfaces are still in development, and they are not fully available yet in the stable versions. 


\begin{tabular}{|l|r|r|r|r|c|}
\hline & LP & CLP(Q) & Tab & Mod TCLP(Q) & Graph \\
\hline Left recursion & - & - & 2311 & $\mathbf{1 2 8 6}$ & \multirow{2}{*}{ Without cycles } \\
Right recursion & $>5$ min. & 5136 & 3672 & $\mathbf{2 2 3 7}$ & \\
\hline Left recursion & - & - & - & $\mathbf{7 4 2}$ & \multirow{2}{*}{ With cycles } \\
Right recursion & - & 10992 & - & 1776 & \\
\hline
\end{tabular}

Table 2: Run time (ms) for dist/3. '-' means no termination.

\begin{tabular}{|l|r|r|r|}
\hline & CLP( $\left.\mathbb{D}_{\leq}\right)$ & Orig. TCLP( $\left.\mathbb{D}_{\leq}\right)$ & Mod TCLP( $\left.\mathbb{D}_{\leq}\right)$ \\
\hline truckload(300) & 40452 & $\mathbf{2 9 0 3}$ & 7268 \\
truckload(200) & 4179 & $\mathbf{1 0 1 5}$ & 2239 \\
truckload(100) & 145 & $\mathbf{1 4 0}$ & 259 \\
\hline step_bound(30) & - & 2657 & $\mathbf{1 4 6 9}$ \\
step_bound(20) & - & 2170 & $\mathbf{1 2 6 7}$ \\
step_bound(10) & - & 917 & $\mathbf{8 4 5}$ \\
\hline
\end{tabular}

Table 3: Performance comparison (ms) of CLP vs original TCLP vs Mod TCLP using $\mathbb{D}_{\leq}$for truckload/4 and step_bound/4. '-' means no termination.

\subsection{The Cost of Modularity: Mod TCLP vs. Original TCLP}

The original TCLP implementation (Chico de Guzmán et al. 2012) was deeply intertwined with the tabling engine and had a comparatively low overhead. Since it was done on the same platform as ours (Ciao Prolog) and shares several components and low-level implementation decisions, it seems a fair and adequate baseline to evaluate the performance cost of the added modularity. We will evaluate both frameworks using exactly the same implementation of difference constraints (Section 5.1) and two benchmarks:

truckload(P, Load, Dest, Time) (Cui and Warren 2000; Schrijvers et al. 2008): it solves a shipment problem given a maximum Load for a truck, a destination Dest, and a list of packages to ship ( 1 to $P$.) We set $P=30$, Dest=chicago and use Load as parameter to vary its complexity. truckload/4 does not need tabling, but tabling speeds it up.

step_bound(Init, Dest, Steps, Limit): it is a left-recursive graph reachability program similar to dist/3 that constrains the total number (Limit) of edge traversals. step_bound/4 needs tabling in the case of graphs with cycles, as it is the case of the graph we will use in this evaluation.

Table 3 shows that truckload/4 incurs a nearly three-fold increase in execution time with respect to the initial non-modular TCLP(D $\left.\mathbb{D}_{\leq}\right)$implementation. This is mainly due to the overhead of the control flow. In the original implementation, execution did not leave the level of $\mathrm{C}$, as the tabling engine called directly the constraint solver, also written in C. However, in Mod TCLP, the tabling engine (in C) calls the interface level (written in Prolog), which calls back the constraint solver (in C). The additional overhead is the price we pay to make it much easier to plug in additional constraint solvers, which in the original TCLP needed ad-hoc, low level wiring.

However, step_bound $/ 4$ is less efficient in the original TCLP( $\left.\mathbb{D}_{\leq}\right)$implementation than in Mod TCLP, and cannot be executed in CLP(D $\left.\mathbb{D}_{\leq}\right)$due to the cycles in the graph. The reason behind this improvement is the enhanced answer management strategy whose 


\begin{tabular}{|l|r|r|r|r|}
\hline & \multicolumn{4}{|c|}{ Mod TCLP(D } \\
\cline { 2 - 5 } & \multicolumn{1}{|c|}{$\emptyset$} & & \multicolumn{1}{c|}{$\rightarrow$} & \multicolumn{1}{c|}{} \\
\hline truckload(300) & 742039 & 7806 & 7780 & $\mathbf{7 2 6 8}$ \\
truckload(200) & 11785 & 2314 & 2354 & $\mathbf{2 2 3 9}$ \\
truckload(100) & 300 & 263 & 263 & $\mathbf{2 5 9}$ \\
\hline step_bound(30) & - & 8450 & - & $\mathbf{1 4 6 9}$ \\
step_bound(20) & - & 6859 & 38107 & $\mathbf{1 2 6 7}$ \\
step_bound(10) & - & 2846 & 8879 & $\mathbf{8 4 5}$ \\
\hline
\end{tabular}

Table 4: Run time (ms) comparison of answer management strategies using Mod TCLP(D $\left.\mathbb{D}_{\leq}\right)$for truckload/4 and step_bound/4.

implementation was made possible by our modular design. We will explore this point in the next section.

\subsection{Improved Answer Management Strategies}

The modular design of Mod TCLP makes it possible to implement more easily hooks for internal operations. In particular, the solver interface can include the answer_compare/3 operation which determines whether a new answer entails, is entailed by, or none of them, some previous answer. This can be used to decide whether to add or not a new answer and remove or not an existing answer. This is undoubtedly expensive in general, but as advanced in Section 4.1, it holds promise for improving performance. To validate this intuition, we executed again truckload/4 and step_bound/4 with TCLP( $\left.\mathbb{D}_{\leq}\right)$under four different answer management strategies:

$\emptyset$ all the answers are stored.

checks if new answers entail previous answers. If so, the new answer is discarded. That is the strategy used in the original TCLP framework.

$\rightarrow$ checks if new answers are entailed by previous answers. If so, the previous answers are flagged as removed and ignored, and the new answer is stored.

$\leftrightarrow$ checks entailment in both directions, discarding new answers and removing more particular answers.

The results in Table 4 confirm that, in the examples studied, and despite the cost of these strategies, the computation time is reduced. The " $\leftrightarrow$ " strategy proves to be the best one, although by a small margin in some cases.

On the other hand, the worst strategy is ' $\theta$ ', which for the truckload/4 program increases the runtime several order of magnitudes for large cases, while for the step_bound/4 program the execution does not terminate because it runs out of memory when trying to generate infinitely many repeated answers. While truckload $/ 4$ behaves similarly for the other strategies, step_bound $/ 4$ varies drastically (i.e., ' $\rightarrow$ ' runs out of memory for the largest case).

Part of the reasons for these differences can be inferred from Table 5, where, for each benchmark and strategy, we show how many of the generated answers were saved, discarded before being inserted, or removed after insertion. Note that these results are independent of the constraint solver used (i.e., executing the same programs using CLP(Q) or CLP $(R)$ instead of $C L P\left(\mathbb{D}_{\leq}\right)$generates the same answers). 


\begin{tabular}{|c|l|r|r|r|r|}
\hline $\begin{array}{c}\text { Answer } \\
\text { strategy }\end{array}$ & & \# Sav. & \# Dis. & \# Rem. & \# Ret. \\
\hline \multirow{3}{*}{$\emptyset$} & truckload(300) & 448538 & 0 & 0 & 14999 \\
& truckload(200) & 52349 & 0 & 0 & 1520 \\
& truckload(100) & 2464 & 0 & 0 & 58 \\
\hline \multirow{6}{*}{} & truckload(300) & 67503 & 9971 & 0 & 41 \\
& truckload(200) & 16456 & 1325 & 0 & 23 \\
& truckload(100) & 1525 & 52 & 0 & 6 \\
\cline { 2 - 6 }$\rightarrow$ & step_bound(30) & 44549 & 716826 & 0 & 252 \\
& step_bound(20) & 37548 & 599259 & 0 & 242 \\
& step_bound(10) & 15625 & 242351 & 0 & 165 \\
\hline \multirow{5}{*}{$\rightarrow$} & truckload(300) & 75272 & 0 & 9460 & 30 \\
& truckload(200) & 17568 & 0 & 1298 & 18 \\
& truckload(100) & 1490 & 0 & 49 & 9 \\
\cline { 2 - 6 } & step_bound(30) & $>1145690$ & 0 & $>1074071$ & - \\
& step_bound(20) & 946309 & 0 & 891078 & 441 \\
& step_bound(10) & 294728 & 0 & 276867 & 221 \\
\hline \multirow{6}{*}{$\leftrightarrow$} & truckload(300) & 48524 & 6596 & 1740 & 5 \\
& truckload(200) & 13550 & 1046 & 240 & 5 \\
& truckload(100) & 1343 & 45 & 10 & 3 \\
\cline { 2 - 6 } & step_bound(30) & 9697 & 74528 & 4571 & 25 \\
& step_bound(20) & 9352 & 71658 & 4371 & 25 \\
& step_bound(10) & 6650 & 56935 & 3019 & 25 \\
\hline
\end{tabular}

Table 5: Number of answers: saved (Sav.), discarded (Dis.), removed (Rem.) and returned to the query (Ret.) for each answer management strategy.
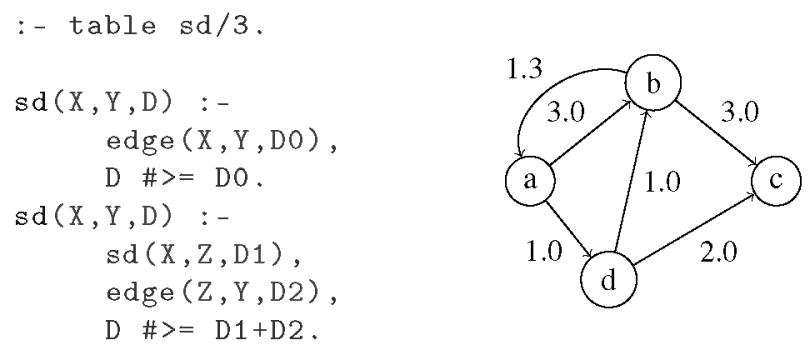

Fig. 10: sd/3 - a shortest-distance program.

For truckload/4, the ' $\rightarrow$ ' and the ' $\leftarrow$ ' strategies generate, discard / remove, and return a similar number of answers, which means that their impact in execution time is not very important. It is notwithstanding interesting to note that there is no slowdown when using the more complex strategy, ' $\leftrightarrow$ '. For step_bound $/ 4$, ' $\rightarrow$ ' generates many more candidate answers than either of the other two - in excess of one million for step_bound (30) but ' $\leftarrow$ ' also generates one order of magnitude more candidates answers than ' $\leftrightarrow$ '. Note that the number of generated answers is not always the same since, as discussed before, fewer saved answers wake up fewer consumers.

As an additional example of the usefulness of obtaining the most general correct answer, Fig. 10 shows a graph and the program sd/3, used in (Cui and Warren 2000) to calculate the "shortest distance" between the nodes in the graph. For a query such as 
?- $s d(X, Y, D i s t)$ the system reported in (Cui and Warren 2000) returns a sequence of $n$ answers of the form Dist \# $>=N_{k}$. Each $N_{k}$ is the current achievable shortest distance from $X$ to $Y$, such as $N_{1}>\cdots>N_{n}$, and the later $N_{n}$ is the shortest distance from $X$ to $Y$. E.g., for the query ?- $\operatorname{sd}(\mathrm{a}, \mathrm{c}, \mathrm{Dist})$ it returns Dist \# $>=6.0$ and Dist \# $>=3.0$. While using Mod TCLP under the ' $\leftrightarrow$ ' strategy the evaluation of the query ?- sd (a, c,Dist) only returns the answer Dist \#>=3.0 (the most general) which corresponds to the tightest bound for the shortest distance between the nodes a and $\mathrm{c}$.

\subsection{Improved Two-Step Projection}

The design of Mod TCLP makes it possible to postpone the projection during the call / answer entailment phase using the Two-Step projection. As we advanced in Section 4.4, it holds promise for performance improvements. To validate this intuition, we use two benchmarks:

$\mathrm{fib}(\mathrm{N}, \mathrm{F})$ the doubly recursive Fibonacci program run backwards. It is well-known that tabling reduces $\mathrm{fib} / 2$ complexity from exponential to linear. In addition, CLP makes it possible to run exactly the same program backwards to find the index of some Fibonacci number by generating a system of equations whose solution is the index of the given Fibonacci number (e.g., for the query ?- $f i b(N, 89)$, the answer is $\mathrm{N}=11$ ). Under CLP, the size of this system of equations grows exponentially with the index of the Fibonacci number. However, under TCLP, entailment makes redundant equations not to be added and solving them becomes less expensive. Additionally, entailment makes it possible to terminate (with failure) even when the query does not contain a non Fibonacci number, e.g., fib $\left(\mathrm{N}, 10^{314}\right)$.

$\operatorname{dist}(X, Y, D)$ the program already used in Section 6.1.

We executed each of them with Mod TCLP(Q) and the two designs for the call projection we discussed earlier:

One-Step: The projection of the call is executed before the call entailment phase (Fig. 9). Note that CLP(Q) does not need this projection to check entailment of the current call constraint store w.r.t. another constraint store.

Two-Step: The projection of the call is executed using final_call_projection/3 and, therefore, it is only executed when the call turns out to be a generator.

The results in Table 6 (top) confirm that, in the examples studied, the Two-Step design reduces the computation time, although only by a small margin in the case of dist/3 with left recursion. That is because, as we see in Table 6 (bottom), using the Two-Step projection, dist $/ 3$ with left recursion executes the projection of a call only once while using One-Step it executes the projection twice and therefore we only save the execution of one projection. Since they are executed early in the evaluation, the constraint store is small and their execution is faster than in the case of dist/ 3 with right recursion. Note that using the Two-Step projection, dist/ 3 with right recursion executes up to 8 times fewer call projections, and as consequence its execution has better performance.

On the other hand, $\mathrm{fib} / 2$ reduces drastically the computation time using Two-Step projection because, during the execution, call entailment is checked many times (although the 


\begin{tabular}{|l|r|r|r|r|}
\hline Run-time (ms) & \multicolumn{3}{|c|}{ Mod TCLP(Q) } \\
\cline { 3 - 5 } & & One-Step & Two-Step & Ratio \\
\hline fib $\left(\mathrm{N}, \mathrm{F}_{1500}\right)$ & 206963 & $\mathbf{1 2 6 4 6 1}$ & 1.63 \\
$\mathrm{fib}\left(\mathrm{N}, \mathrm{F}_{1000}\right)$ & 89974 & $\mathbf{5 5 1 8 3}$ & 1.63 \\
fib $\left(\mathrm{N}, \mathrm{F}_{500}\right)$ & 22133 & $\mathbf{1 3 6 1 2}$ & 1.63 \\
fib $\left(\mathrm{N}, 10^{314}\right)$ & & 205638 & $\mathbf{1 2 5 6 7 0}$ & 1.63 \\
\hline \multirow{2}{*}{ dist/3 right rec. } & Without cycles & 2855 & $\mathbf{2 5 0 6}$ & 1.14 \\
& With cycles & 2399 & $\mathbf{1 8 5 0}$ & 1.30 \\
\hline \multirow{2}{*}{ dist/3 left rec. } & Without cycles & 1436 & $\mathbf{1 4 2 8}$ & 1.01 \\
& With cycles & 776 & $\mathbf{7 7 2}$ & 1.01 \\
\hline
\end{tabular}

\begin{tabular}{|l|r|r|r|r|}
\hline \# call projections & \multicolumn{3}{|c|}{ Mod TCLP(Q) } \\
\cline { 3 - 5 } & & One-Step & Two-Step & Ratio \\
\hline fib $\left(N, F_{1500}\right)$ & 1129497 & $\mathbf{5 6 5 5 0 0}$ & 2.00 \\
fib $\left(N, F_{1000}\right)$ & 502997 & $\mathbf{2 5 2 0 0 0}$ & 2.00 \\
fib $\left(N, F_{500}\right)$ & 126497 & $\mathbf{6 3 5 0 0}$ & 1.99 \\
fib $\left(N, 10^{314}\right)$ & & 1126499 & $\mathbf{5 6 3 2 5 2}$ & 2.00 \\
\hline \multirow{2}{*}{ dist/3 right rec. } & Without cycles & 1563 & $\mathbf{1 8 1}$ & 8.64 \\
& With cycles & 2144 & $\mathbf{4 4 3}$ & 4.84 \\
\hline \multirow{2}{*}{ dist/3 left rec. } & Without cycles & 2 & $\mathbf{1}$ & 2.00 \\
& With cycles & 2 & $\mathbf{1}$ & 2.00 \\
\hline
\end{tabular}

Table 6: Run time in ms (top) and number of call projections (bottom) for each projection design, One-Step and Two-Step.

Note: $F_{n}$ is the $n^{\text {th }}$ Fibonacci number, and $10^{314}$ is not a Fibonacci number.

ratio of useless projection is similar to that of dist/3 with left recursion). Note that the Fibonacci number $F_{1500}$ and $10^{314}$ have the same size ( 315 digits) and the run time / number of projections for $\mathrm{fib}\left(\mathrm{N}, \mathrm{F}_{1500}\right)$ and for $\mathrm{fib}\left(\mathrm{N}, 10^{314}\right)$ are similar. That is because the work needed to find the index of a Fibonacci number is similar to the work needed to confirm whether a number is or not a Fibonacci number.

\subsection{Abstract Interpretation: Tabling vs. TCLP(Lat)}

We compare here tabling and TCLP using two versions of a simple abstract interpreter (Cousot and Cousot 1977). The interpreter executes the programs to be analyzed on an abstract domain, collecting the possible values at every point until a fixpoint is reached. The result of the execution is a safe approximation of the run-time values of the variables in the concrete domain. The abstract domain we have used in this example is the signs abstract domain (Fig. 11).

The two versions of the abstract interpreter we have used are:

Tabling This version is a simple abstract interpreter written using tabling. This ensures termination, as the abstract domain is finite.

TCLP This version is based on the previous abstract interpreter, but it uses the TCLP(Lat) constraint solver interface (Section 5.2) to operate on the abstract domain and set up constraints over the variables. The main differences with the tabling version is that TCLP uses constraint entailment instead of variant checking for loop detection and, 


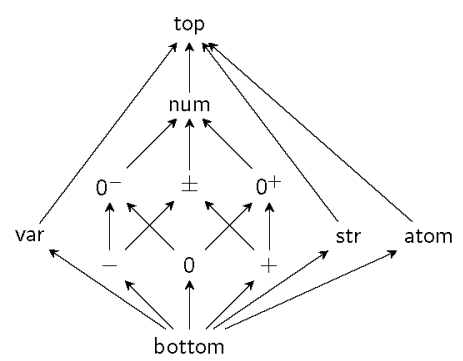

Fig. 11: Signs abstract domain.

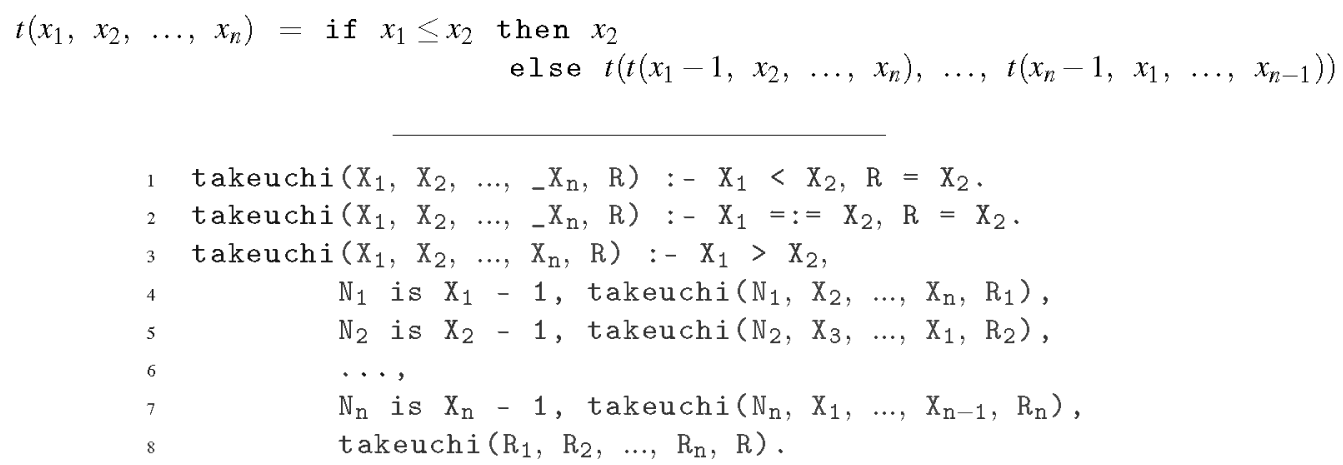

Fig. 12: $n$-dimensional Takeuchi function and its implementation.

therefore, it can also use the answers to more general goals to avoid computing more particular goals.

We applied our abstract interpreter to two programs:

takeuchi/m (Fig. 12): a Prolog implementation of the $n$-dimensional generalization of the Takeuchi function (Knuth 1991). The program is parametric on the number of input arguments $n$ and it returns the result in its last argument.

sentinel/m (Fig. 13): a variant of a synthetic program presented in (Genaim et al. 2001). It receives as input its first argument (the Sentinel) and the next $n$ arguments $A_{1}, \ldots, A_{n}$ are a ring-ordered ${ }^{11}$ series of numbers. The outputs are the arguments $B_{1}, \ldots, B_{n}$, which correspond to a circular shift of $A_{1}, \ldots, A_{n}$ such that on success $\mathrm{B}_{\mathrm{i}}<\mathrm{B}_{\mathrm{i}+1}$ for all $i<n$ and: if Sentinel $=0$, the first half of $\mathrm{B}_{\mathrm{i}}$ are negative and the second half are positive; if Sentinel $<0, \mathrm{~B}_{\mathrm{i}}<$ Sentinel for all $i$; and if Sentinel $>0, \mathrm{~B}_{\mathrm{i}}>$ Sentinel for all $i$.

Table 7 shows the run time results of analyzing takeuchi/m parameterized by the dimension of the function, $n(m=n+1)$, and sentinel/m parameterized by $n$, the length of the ring $(m=2 n+1)$. In both examples the analysis with the TCLP version of the interpreter is faster than the analysis with the interpreter without constraints: the latter has

${ }^{11}$ I.e., there is a $j$ such that $A_{j}<A_{j+1}, A_{j+1}<A_{j+2}, \ldots, A_{n}<A_{1}, A_{1}<A_{2}, \ldots, A_{j-2}<A_{j-1}$. 


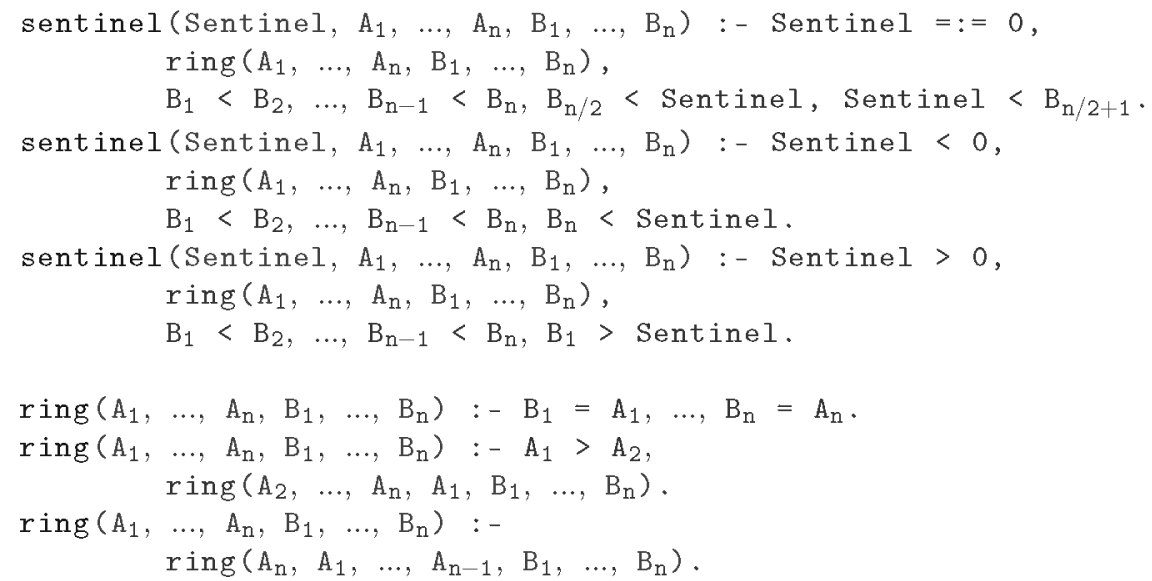

Fig. 13: sentinel/m program.

\begin{tabular}{|l|l|r|r|}
\hline & & Tabling & Mod TCLP(Lat $)$ \\
\hline \multirow{3}{*}{ takeuchi/m $(m=n+1)$} & $\mathrm{n}=8$ & 31.44 & $\mathbf{8 . 0 9}$ \\
& $\mathrm{n}=6$ & 13.75 & $\mathbf{5 . 8 5}$ \\
& $\mathrm{n}=3$ & $\mathbf{2 . 4 2}$ & 3.12 \\
\hline \multirow{3}{*}{ sentinel/m $(m=2 n+1)$} & $\mathrm{n}=8$ & 1375.13 & $\mathbf{9 . 2 3}$ \\
& $\mathrm{n}=6$ & 218.93 & $\mathbf{6 . 5 3}$ \\
& $\mathrm{n}=4$ & 30.99 & $\mathbf{4 . 5 6}$ \\
\hline
\end{tabular}

Table 7: Run time (ms) for analyze (takeuchi/m) and for analyze (sentinel/m).

to evaluate each permutation completely in the recursive predicates, while the former can suspend and save computation time using results from a previous, more general, call.

Let us examine an example. For a variable $\mathrm{A}$, let us write $\mathrm{A}^{a b s}$ to represent $\mathrm{A} \sqsubseteq a b s$. On the one hand, when an initial goal ring $\left(\mathrm{A}_{1}{ }^{t o p}, \ldots, \mathrm{A}_{\mathrm{n}}{ }^{t o p}, \mathrm{~B}_{1}{ }^{t o p}, \ldots, \mathrm{B}_{\mathrm{n}}{ }^{t o p}\right)$ is interpreted by the TCLP analyzer, the first clause of $\mathrm{ring} / 2 \mathrm{n}$ produces the first answer. Then the interpreter continues with the second clause, interprets the goal $A_{1}>A_{2}$ and starts the evaluation of ring $\left(\mathrm{A}_{2}{ }^{n u m}, \ldots, \mathrm{A}_{\mathrm{n}}{ }^{\text {top }}, \mathrm{A}_{1}{ }^{\text {num }}, \mathrm{B}_{1}{ }^{\text {top }}, \ldots, \mathrm{B}_{\mathrm{n}}{ }^{\text {top }}\right)$. Since num $\sqsubseteq$ top , this new call entails the previous one and TCLP suspends this execution. Then the interpreter continues with the third clause, which starts the evaluation of $\operatorname{ring}\left(\mathrm{A}_{\mathrm{n}}{ }^{t o p}, \mathrm{~A}_{1}{ }^{t o p}, \ldots, \mathrm{A}_{\mathrm{n}-1}{ }^{t o p}, \mathrm{~B}_{1}{ }^{\text {top }}, \ldots, \mathrm{B}_{\mathrm{n}}{ }^{\text {top }}\right)$, and TCLP also suspends the execution. Since the generator does not have more clauses to evaluate, TCLP resumes the suspended execution with the previously obtained answer. Each consumer produces a new answer but since they are at least as particular as the previous one, they are discarded.

On the other hand when the initial goal is evaluated in the tabling interpreter with $\mathrm{A}_{1}{ }^{t o p}, \ldots, \mathrm{A}_{\mathrm{n}}{ }^{t o p}, \mathrm{~B}_{1}{ }^{t o p}, \ldots, \mathrm{B}_{\mathrm{n}}{ }^{t o p}$ as entry substitution, the first answer is also produced. Then the interpreter continues with the second clause, interprets the goal $A_{1}>A_{2}$ and starts the evaluation of the recursive call with the entry substitution $\mathrm{A}_{1}{ }^{\text {num }}, \ldots, \mathrm{A}_{\mathrm{n}}{ }^{\text {top }}, \mathrm{B}_{1}{ }^{\text {num }}, \ldots, \mathrm{B}_{\mathrm{n}}{ }^{\text {top }}$. However, tabling does not suspend the execution because it is not a variant call of the previous one, which results in increased computation time.

Table 8 shows the results of analyzing sentinel/m in two different scenarios: with- 


\begin{tabular}{|l|r|r|r|r|r|}
\hline \multicolumn{2}{|c|}{} & \multicolumn{2}{|c|}{ Tabling } & \multicolumn{2}{c|}{ Mod TCLP(Lat) } \\
\cline { 3 - 6 } \multicolumn{2}{|c|}{} & $\begin{array}{c}\text { constraints } \\
\text { before call }\end{array}$ & $\begin{array}{c}\text { unconstrained } \\
\text { call }\end{array}$ & $\begin{array}{c}\text { constraints } \\
\text { before call }\end{array}$ & $\begin{array}{c}\text { unconstrained } \\
\text { call }\end{array}$ \\
\hline \multirow{2}{*}{ sentinel/m } & $\mathrm{n}=8$ & $\mathbf{7 4 9 . 3 8}$ & 1375.13 & $\mathbf{5 . 2 9}$ & 9.23 \\
$(m=2 n+1)$ & $\mathrm{n}=6$ & $\mathbf{9 8 . 8 0}$ & 218.93 & $\mathbf{3 . 3 1}$ & 6.53 \\
& $\mathrm{n}=4$ & $\mathbf{6 . 5 3}$ & 30.99 & $\mathbf{2 . 8 5}$ & 4.56 \\
\hline
\end{tabular}

Table 8: Run time (ms) for analyze (sentinel/m).

out any constraints in the abstract substitution of the variables or adding the constraint Sentine $\sqsubseteq+$ before the analysis. Adding that domain restriction reduces analysis times by approximately the same ratio in both cases. Note that this compares two scenarios which are possible both for TCLP and for tabling without constraints. Additionally, the TCLPbased analyzer would be able to take into account constraints among variables, which would not be directly possible using tabling without constraints.

\section{Related Work}

The initial ideas of tabling and constraints originate in (Kanellakis et al. 1995), where a variant of Datalog featuring constraints was proposed. The time and space properties associated with the bottom-up evaluation of Datalog were studied in (Toman 1997b), where a top-down evaluation strategy featuring tabling was proposed.

XSB (Swift and Warren 2012) was the first logic programming system that provided tabled CLP as a generic feature, instead of resorting to ad-hoc adaptations. This was done by extending XSB with attributed variables (Cui and Warren 2000), one of the most popular mechanism to implement constraint solvers in Prolog. However, one of its drawbacks is that it only uses variant call checking (even for goals with constraints), instead of entailment checking of calls / answers. This makes programs terminate in fewer cases than using entailment and takes longer in other cases. This is similar to what happens in tabled logic programs with and without subsumption (Swift and Warren 2010). From the point of view of interfacing / adding additional CLP solvers to existing systems, the framework in (Cui and Warren 2000) requires the constraint solver to provide the predicates projection/1 and entail/2, which are used to discard more particular answers, but only in one direction. It also requires the implementation of the predicate abstract/3, which has to take care of the call abstraction. However, it is not clear if this predicate is part of the constraint solver or of the user program.

A general framework for CHR under tabled evaluation is described in (Schrijvers et al. 2008). It takes advantage of the flexibility that CHR provides for writing constraint solvers, but it also lacks call entailment checking and enforces total call abstraction: all constraints are removed from calls before executing them, which can result in non-termination w.r.t. systems which use entailment. Besides, the need to change the representation between CHR and Herbrand terms takes a toll in performance. From the interface point of view, the framework provides interesting hooks: projection(PredName) specifies that predicate PredName/1 determines how projection is to be performed, which makes it possible to, for example, ignore arguments; canonical_form (PredName) modifies the answer store to a canonical form as defined by PredName/2, so that identical answers can be detected 
(e.g. using sort $/ 2$ the constraints $[\operatorname{leq}(1, X), \operatorname{leq}(X, 3)]$ and $[\operatorname{leq}(X, 3), \operatorname{leq}(1, X)]$ are reduced to the same canonical form); and answer_combination (PredName), if specified, applies PredName/ 3 in such a way that two answers can be merged into one.

Failure Tabled CLP (Gange et al. 2013) implements a verification-oriented system which has several points in common with TCLP. Interestingly, it can learn from failed derivations and uses interpolants instead of constraint projection to generate conditions for reuse. It will however not terminate in some cases even with the addition of counters to implement a mechanism akin to iterative deepening.

Last, the original TCLP proposal (Chico de Guzmán et al. 2012) features entailment checking for calls and (partially) for answers, executes calls with all the constraints, and has good performance. However, from the interface point of view, it did not clearly state which operations must be present in the constraint solver, which made it difficult to extend, and was not focused on a modular design which, for example, made implementing specific answer management strategies cumbersome.

\section{Conclusions and Further Work}

We have presented an approach to include constraint solvers in logic programming systems with tabling. Our main goal is making the addition of new constraint solvers easier while taking full advantage of entailment between constraint stores. In order to achieve this, we determined the services that a constraint solver should provide to a tabling engine. This interface has been designed to give the constraint solver freedom to implement them. To validate our design, we have interfaced one solver previously written in $\mathrm{C}\left(\mathrm{CLP}\left(\mathbb{D}_{\leq}\right)\right)$, two existing classical solvers (CLP(Q/R)), and a new solver (CLP(Lat)), and we have found the integration to be easy - certainly easier than with other designs - , validating the usefulness of the capabilities that our system provides.

We evaluated its performance in a series of benchmarks. In some of them large savings are attained w.r.t. non-tabled/tabled executions, even taking into account the penalty to pay for the additional flexibility and modularity. We are in any case confident that there is still ample space to improve the efficiency of the implementation, since in the current implementation we gave more importance to the cleanliness of the code and the design.

The facilities that our framework provides to integrate constraint solvers with tabling pave the way to new research directions:

- Explore richer, faster, and more flexible implementations of abstract interpretationbased analyzers.

- Evolve CLP(Lat) into a lattice domain that can capture reasoning in ontologies and explore its usage to implement constraint tabled-based reasoning systems featuring automatic reuse of more general concepts and combinations of answers into more general concepts.

- Implement a TCLP interface for a constraint solver over finite domains, CLP(FD) (Van Hentenryck 1989; Dincbas et al. 1988; Díaz and Codognet 1993). CLP(FD) is widely used to model discrete problems such as scheduling, planning, packing, and timetabling. The implementation is not straightforward due to the difficulty of expressing projection and entailment inside CLP(FD) (Carlson et al. 1994). 


\section{References}

Carlson, B., Carlsson, M., And Diaz, D. 1994. Entailment of Finite Domain Constraints. In International Conference on Logic Programming (ICLP'94). The MIT Press.

Charatonik, W., Mukhopadhyay, S., And Podelski, A. 2002. Constraint-Based Infinite Model Checking and Tabulation for Stratified CLP. In ICLP'O2, P. J. Stuckey, Ed. Lecture Notes in Computer Science, vol. 2401. Springer, 115-129.

Chico de Guzmán, P., Carro, M., Hermenegildo, M. V., and S tuckey, P. 2012. A General Implementation Framework for Tabled CLP. In FLOPS'12, T. Schrijvers and P. Thiemann, Eds. Number 7294 in LNCS. Springer Verlag, 104-119.

Cousot, P. AND Cousot, R. 1977. Abstract Interpretation: a Unified Lattice Model for Static Analysis of Programs by Construction or Approximation of Fixpoints. In ACM Symposium on Principles of Programming Languages (POPL'77). ACM Press, 238-252.

CUI, B. AND WARREN, D. S. 2000. A system for Tabled Constraint Logic Programming. In Computational Logic. 478-492.

Dawson, S., Ramakrishnan, C. R., And Warren, D. S. 1996. Practical Program Analysis Using General Purpose Logic Programming Systems - A Case Study. In Proceedings of the ACM SIGPLAN'96 Conference on Programming Language Design and Implementation. ACM Press, New York, USA, 117-126.

Díaz, D. And Codognet, P. 1993. A Minimal Extension of the WAM for $c l p(f d)$. In Proceedings of the Tenth International Conference on Logic Programming. Budapest, MIT press, 774-790.

Dincbas, M., Hentenryck, P. V., Simonis, H., And Aggoun, A. 1988. The Constraint Logic Programming Language CHIP. In Proceedings of the 2 nd International Conference on Fifth Generation Computer Systems. 249-264.

Falaschi, M., Levi, G., Martelli, M., And Palamidessi, C. 1989. Declarative Modeling of the Operational Behaviour of Logic Programs. Theoretical Computer Science 69, 289-318.

Frigioni, D., MarchetTi-SpaCCAmela, A., And NAnni, U. 1998. Fully Dynamic Shortest Paths and Negative Cycles Detection on Digraphs with Arbitrary Arc Weights. In ESA. 320-331.

Gange, G., Navas, J. A., Schachte, P., Søndergaard, H., and S tuckey, P. J. 2013. Failure Tabled Constraint Logic Programming by Interpolation. TPLP 13, 4-5, 593-607.

Genaim, S., Codish, M., And Howe, J. 2001. Worst-Case Groundness Analysis Using Definite Boolean Functions. Theory and Practice of Logic Programming 1, 05, 611-615.

Hermenegildo, M. V., Bueno, F., Carro, M., López, P., Mera, E., Morales, J., And Puebla, G. 2012. An Overview of Ciao and its Design Philosophy. Theory and Practice of Logic Programming 12, 1-2 (January), 219-252. http://arxiv.org/abs/1102.5497.

Holzbaur, C. 1995. OFAI CLP(Q,R) Manual, Edition 1.3.3. Tech. Rep. TR-95-09, Austrian Research Institute for Artificial Intelligence, Vienna.

JAfFAR, J. AND Maher, M. 1994. Constraint Logic Programming: A Survey. Journal of Logic Programming 19/20, 503-581.

Kanellakis, P. C., Kuper, G. M., And Revesz, P. Z. 1995. Constraint Query Languages. J. Comput. Syst. Sci. 51, 1, 26-52.

Knuth, D. E. 1991. Textbook Examples of Recursion. Artificial Intelligence and Mathematical Theory of Computation: Papers in Honor of John McCarthy, 207-230.

Marriott, K. AND STUCKey, P. J. 1998. Programming with Constraints: an Introduction. MIT Press.

Ramakrishna, Y., Ramakrishnan, C., Ramakrishnan, I., Smolka, S., Swift, T., And WARreN, D. 1997. Efficient Model Checking Using Tabled Resolution. In Computer Aided Verification. Lecture Notes in Computer Science, vol. 1254. Springer Verlag, 143-154.

Ramakrishnan, I., RaO, P., Sagonas, K., Swift, T., and Warren, D. 1995. Efficient Tabling Mechanisms for Logic Programs. In ICLP'95. 697-711. 
SChriJVers, T., Demoen, B., AND WARREN, D. S. 2008. TCHR: a Framework for Tabled CLP. TPLP 8, 4, 491-526.

SWIFT, T. AND WARREN, D. S. 2010. Tabling with answer subsumption: Implementation, applications and performance. In $J E L I A$, T. Janhunen and I. Niemelä, Eds. Lecture Notes in Computer Science, vol. 6341. Springer, 300-312.

SwifT, T. And Warren, D. S. 2012. XSB: Extending Prolog with Tabled Logic Programming. TPLP 12, 1-2, 157-187.

TAMAKI, H. AND SATO, M. 1986. OLD Resolution with Tabulation. In Third International Conference on Logic Programming. Lecture Notes in Computer Science, Springer-Verlag, London, 84-98.

Toman, D. 1997a. Constraint Databases and Program Analysis Using Abstract Interpretation. In Constraint Databases and Their Applications. Lecture Notes in Computer Science, vol. 1191. $246-262$.

Toman, D. 1997b. Memoing Evaluation for Constraint Extensions of Datalog. Constraints 2, 3/4, 337-359.

Van Hentenryck, P. 1989. Constraint Satisfaction in Logic Programming. MIT Press.

WArren, D. S. 1992. Memoing for Logic Programs. Communications of the ACM 35, 3, 93-111.

Warren, R., Hermenegildo, M., AND Debray, S. K. 1988. On the Practicality of Global Flow Analysis of Logic Programs. In Fifth International Conference and Symposium on Logic Programming. MIT Press, 684-699.

Zou, Y., Finin, T., And Chen, H. 2005. F-OWL: An Inference Engine for Semantic Web. In Formal Approaches to Agent-Based Systems. Lecture Notes in Computer Science, vol. 3228. Springer Verlag, 238-248. 


\title{
Description, Implementation, and Evaluation of a Generic Design for Tabled CLP
}

\author{
Joaquín Arias ${ }^{1,2}$ \\ Manuel Carro ${ }^{1,2}$ \\ joaquin.arias@imdea.org, manuel.carro@\{imdea.org,upm.es\} \\ ${ }^{1} I M D E A$ Software Institute, ${ }^{2}$ Universidad Politécnica de Madrid
}

\section{Appendix A CLP Trees and TCLP Forests}

Prolog and CLP follow a depth-first search strategy with chronological backtracking. The computation rule selects constraints and literals from the resolvent from left to right. Literals are resolved against the clauses of the program, selected from top to bottom. When a literal unifies with a clause head, it is substituted by the body of the clause after applying the unifier obtained from the literal-head unification. If a de rivation branch fails because there are no more matching clauses or the constraint store is inconsistent, the evaluation backtracks to the youngest literal that has a candidate matching clause. Depth-first search is incomplete and in general not all answers can be computed. Moreover, there are programs with finite derivations for which logically equivalent programs produce infinite derivations. The use of TCLP can work around this issue in many cases.

We will show the CLP trees and the TCLP forests for the query ?- D \#< 150 , $\operatorname{dist}(a, Y, D)$. for two logically equivalent versions of the dist/3 program: with left recursion (Fig. ??, right) and with right recursion (Fig. ??, right). We use the graph in Fig. A 1, where the length of one of the edges is defined with constraints.
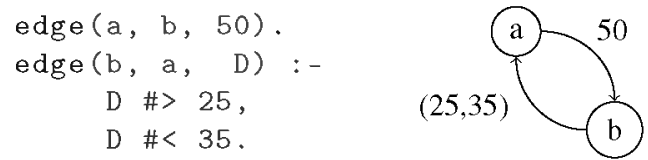

Fig. A 1: Graph definition. $(25,35)$ is the open interval from 25 to 35.

Fig. A 2 and Fig. A 3 (top) are the CLP trees of the right- / left-recursive programs respectively. Fig. A 3 (bottom) and Fig. A 4 are the TCLP forest of the left- / right-recursive programs respectively. In these figures, the nodes of the trees represent the states (Def. ??) of the computation. A state is a tuple $\langle R, c\rangle$, where $R$ is a sequence of goals, $\left[g_{1}, g_{2}, \ldots, g_{n}\right]$ and $c$ is a conjunction of constraints. The numbers attached to each state indicates the order in which they are created.

On the one hand, Fig. A 2 shows a finite CLP tree which finds all the answers and Fig. A 3 (top) shows an infinite CLP tree caused by the left recursion. On the other hand, Fig. A 
3 (bottom) and Fig. A 4 show that the TCLP forest for both programs are finite and all the answers to the query are found, since the use of tabling makes it terminate with left recursion as well.

\section{A.I CLP Tree of dist/3 with Right Recursion}

Fig. A 2 shows the CLP tree of the query using the version of dist/3 with right recursion (Fig. ??, right). We see that the evaluation of the recursive clause generates similar states (s1, s4, s7 and s10), but in each iteration the domain of the constrained variable $\mathrm{D} 2_{i}$ is reduced. As a consequence, the constraint store in state s13 is inconsistent and the evaluation of this derivation fails. The pending branches are evaluated upon backtracking. We explain now how we obtain some of the states; the rest are obtained similarly, so we will skip them:

s1 the initial state is the representation of the query.

$\mathbf{s} 2 \mathrm{i} / \mathbf{i i}$ are obtained by resolving the literal $\operatorname{dist}(\mathrm{a}, \mathrm{Y}, \mathrm{D})$ against the two clauses of the program. The constraints $\mathrm{Y}_{1}=\mathrm{Y} \wedge \mathrm{D}_{1}=\mathrm{D}$ are added to the constraint store.

$\mathbf{s} 3$ is obtained from the leftmost state s2i by adding the constraints of the resolvent $\left[D 1_{1} \#>0, D 22_{1} \#>0, D_{1} \#=D 1_{1}+D 2_{1}\right]$ to the constraint store.

$\mathbf{s 4}$ is obtained by resolving the literal edge $\left(a, Z_{1}, D 1_{1}\right)$. The constraint $\mathrm{Z}_{1}=\mathrm{b} \wedge \mathrm{D} 1_{1}=50$ reduces the domain ${ }^{1}$ of $\mathrm{D} 2_{1}$ to $\mathrm{D} 2_{1}>0 \wedge \mathrm{D} 2_{1}<100$.

s7 is obtained by resolving the literal edge $\left(b, Z_{2}, D 1_{2}\right)$. The constraint $\mathrm{Z}_{2}=\mathrm{a} \wedge \mathrm{D} 1_{2}>25 \wedge \mathrm{D} 1_{2}<35$ reduces the domain of $\mathrm{D} 2_{2}$ to $\mathrm{D} 2_{2}>0 \wedge \mathrm{D} 2_{2}<75$.

s10 is obtained by resolving the literal edge $\left(a, Z_{3}, D 1_{3}\right)$. The constraint $\mathrm{Z}_{3}=\mathrm{b} \wedge \mathrm{D} 1_{3}=50$ reduces the domain of $\mathrm{D} 2_{3}$ to $\mathrm{D} 2_{3}>0 \wedge \mathrm{D} 2_{3}<25$.

$\mathbf{s 1 3}$ is obtained by resolving the literal edge $\left(b, Z_{4}, D 1_{4}\right)$. The constraint $\mathrm{Z}_{4}=\mathrm{a} \wedge \mathrm{D} 1_{4}>25 \wedge \mathrm{D} 1_{4}<35$ is inconsistent with the current constraint store, $\mathrm{D}<150 \wedge \mathrm{D}>125+\mathrm{D} 1_{4}+\mathrm{D} 2_{4} \wedge \mathrm{D} 2_{4}>0 \wedge \ldots$ Its child is a fail node.

s14 is obtained, upon backtracking to the state s11b by resolving the literal edge $\left(b, Y, D 2_{3}\right)$. However, it is also a failed derivation because the resulting constraint store is inconsistent.

$\mathbf{s 1 5}$ is a final state of a successful derivation, obtained upon backtracking to the state s8b by resolving the literal edge $\left(a, Y, D 2_{2}\right)$. The constraint $\mathrm{Y}=\mathrm{a} \wedge \mathrm{D} 2_{3}>25 \wedge$ $\mathrm{D} 2_{3}<35$ is consistent with the constraint store.

a1 is the first answer $\mathrm{Y}=\mathrm{a} \wedge \mathrm{D}>125 \wedge \mathrm{D}<315$, projected onto the variables of the query $(\operatorname{vars}(Q)=\{\mathrm{Y}, \mathrm{D}\})$.

s16 is a final state obtained upon backtracking to the state s5b.

a2 is the second answer, $\mathrm{Y}=\mathrm{a} \wedge \mathrm{D}>75 \wedge \mathrm{D}<85$.

s17 is a final state obtained upon backtracking to the state s2ii.

$\mathbf{a} 3$ is the third and last answer, $\mathrm{Y}=\mathrm{b} \wedge \mathrm{D}=50$

\footnotetext{
${ }^{1}$ We are considering a linear constraint solver over the rational numbers which from $\mathrm{D}<150 \wedge \mathrm{D}=\mathrm{D} 1_{1}+\mathrm{D} 2_{1} \wedge \mathrm{D} 1_{1}=50$ it infers that $\mathrm{D} 2_{1}<100$
} 


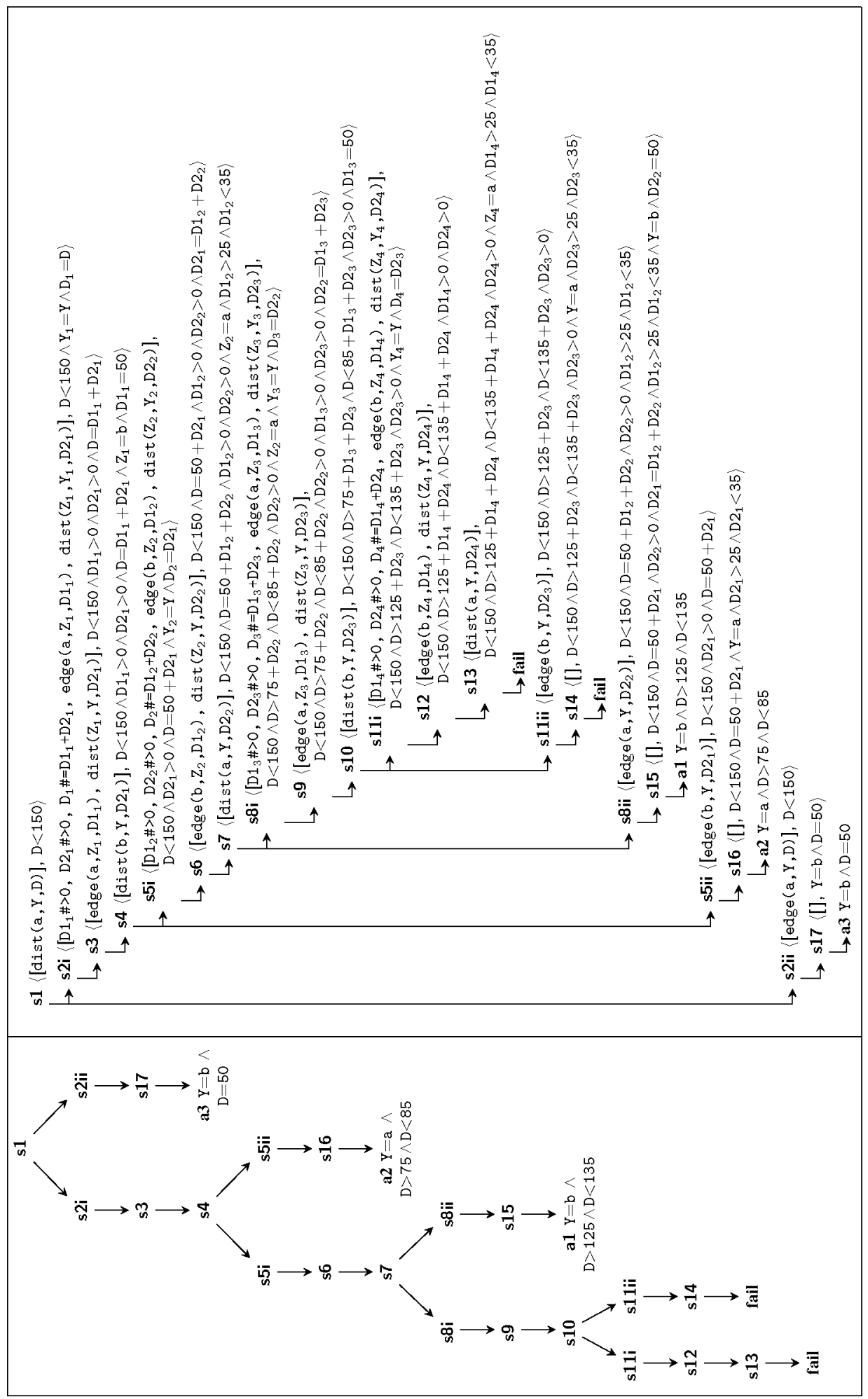

Fig. A 2: CLP tree of the query ?- D \#<150, $\operatorname{dist~}(a, Y, D)$ for dist $/ 3$ with right recursion 


\section{A.2 CLP Tree of dist/3 with Left Recursion}

Fig. A 3 (top) shows the CLP tree of the query to dist/3 with left recursion (Fig. ??, right). We see that the recursive clause also generates similar states $(s 1, s 3, s 5, \ldots)$ but in this example the domain of the constrained variable $D 1_{i}$ remains unchanged, and the evaluation therefore enters a loop. As before, we only explain how we obtain some of the states:

$\mathbf{s} 3$ is obtained from the leftmost state $s 2 \mathrm{i}$. The domain of $\mathrm{D} 1_{1}$ is $\mathrm{D} 1_{1}>0 \wedge \mathrm{D} 1_{1}<150$.

$\mathbf{s 5}$ is obtained from the leftmost node $s 4$. The domain of $\mathrm{D} 1_{2}$ is $\mathrm{D} 1_{2}>0 \wedge \mathrm{D} 1_{2}<150$.

... the evaluation enters a loop.

Although the program that generates this CLP tree is logically equivalent to the previous one, this tree is infinite and no answers are found.

\section{A.3 TCLP Forest of dist/3 with Left Recursion}

Fig. A 3 (bottom) shows the TCLP forest for the query we have been using to the dist/3 program written using left recursion (Fig. ??, right) where the set of tabled predicates is $T a b_{P}=\{$ dist $/ 3\}$. The main point is that at state $\mathrm{s} 3$ the tabling engine detects that the evaluation of $\left\langle\operatorname{dist}\left(a, Z_{1}, D 1_{1}\right), D 1_{1}>0 \wedge D 1_{1}<150\right\rangle$ entails the generator $\langle\operatorname{dist}(a, V 0, V 1)$, $\mathrm{V} 1<150\rangle$ and therefore it suspends the execution and waits until another generator feeds the suspended goal with answers. The evaluation of the state s2ii generates the first answer a1 upon backtracking. Then, the tabling engine resumes the consumer with a 1 and generates a 2 which is used to generate a3. Finally, the evaluation fails after consuming a3 and, since all the clauses have been evaluated and there are no more consumers to be resumed or answers to be consumed, the generator is marked as complete and all the answers are returned. We explain below how some of the states are obtained. The rest of the states are obtained similarly, so we skip them for brevity:

s0 We omit the representation of the TCLP tree for the query $\tau_{P}(\operatorname{dist}(\mathrm{a}, \mathrm{Y}, \mathrm{D}), \mathrm{D}<150)$ and its answer resolution.

s1 the initial state of the TCLP tree $\tau_{P}\left(\operatorname{dist}\left(\mathrm{a}, \mathrm{VO}_{1}, \mathrm{V1} 1_{1}\right), \mathrm{V} 1_{1}<150\right)$ is the renamed generator. (Def. ??).

s2i/ii are obtained by resolving the literal dist (a, Vo, V1) against the two clauses of the program.

$\mathbf{s} 3$ is obtained from the leftmost state s2i by adding the constraints to the constraint store as in the CLP tree.

Ans(s1) the tabled literal $\operatorname{dist}\left(\mathrm{a}, \mathrm{Z}_{1}, \mathrm{D} 1_{1}\right)$ has to be resolved by answer resolution (Def. ??) using the answer from the current TCLP tree $\tau_{P}\left(\operatorname{dist}\left(\mathrm{a}, \mathrm{VO}_{1}, \mathrm{~V} 1_{1}\right), \mathrm{V} 1_{1}<150\right)$ because, after renaming, the projection of the current constraint store onto the variables of the literal entails the projected constraint store of the generator: $\mathrm{V} 1_{1}>0 \wedge \mathrm{V} 1_{1}<150 \sqsubseteq \mathrm{V} 1_{1}<150$. Since the current TCLP tree is under construction and depends on itself, this branch derivation is suspended.

$\mathbf{s 4}$ is a final state of a successful derivation. It is obtained, upon backtracking to the state s2ii, by resolving with edge $(a, v 0, V 1)$. The equations $v 0=b \wedge v 1=50$ are consistent with the constraint store. 


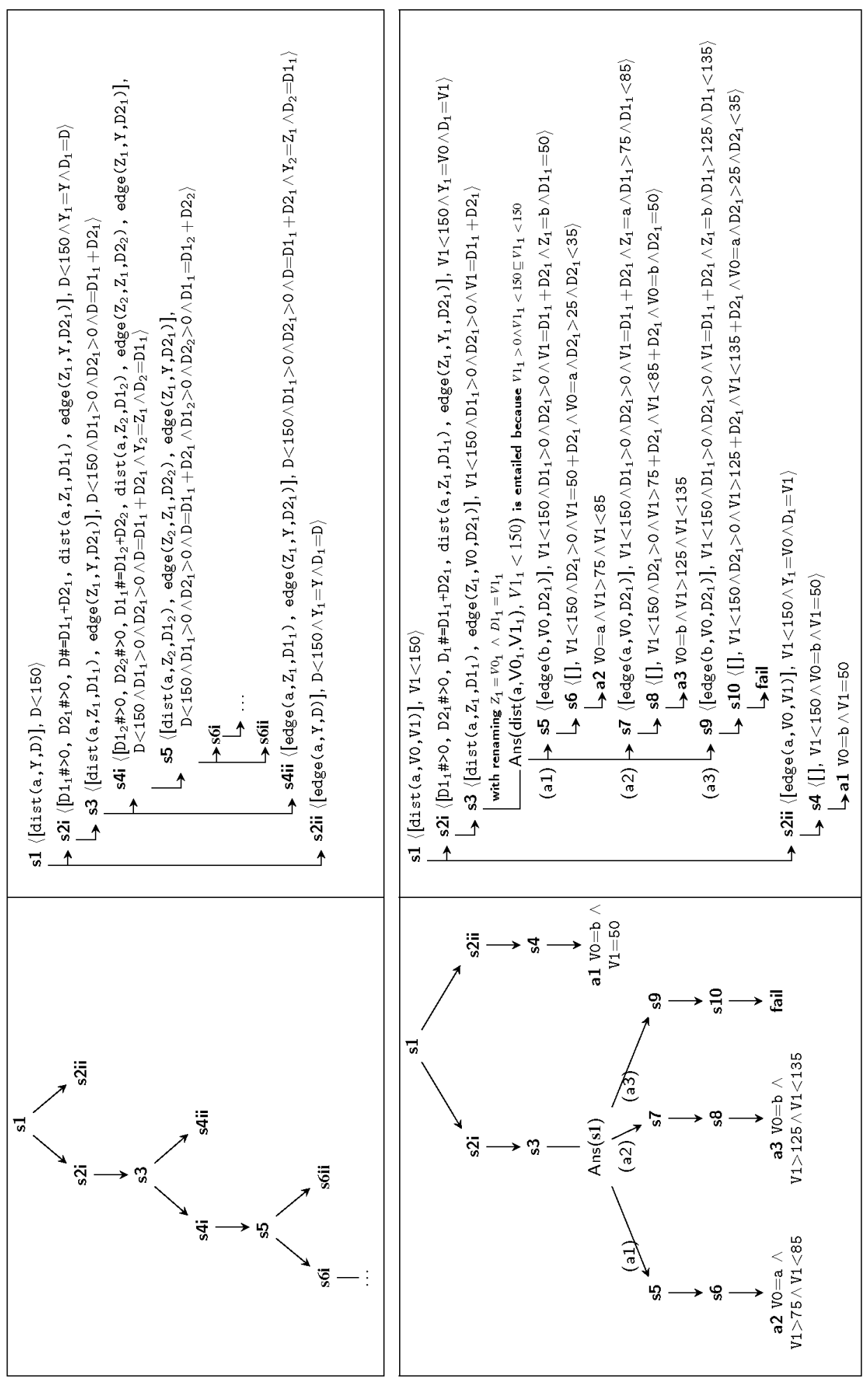

Fig. A 3: CLP tree (top) and TCLP-forest (bottom) of the query ?- $\mathrm{D} \#<150$, dist $(\mathrm{a}, \mathrm{Y}, \mathrm{D})$ for dist/3 with left recursion 
a1 is the first answer, $\mathrm{V} 0=\mathrm{b} \wedge \mathrm{V} 1=50$ (Def. ??). Since is the first one, it is also the more general one.

s5 is obtained from the state s3 (because there are no more branches) by answer resolution consuming a1 (Def. ??).

s6 is a final state obtained by resolving the literal edge ( $\left.b, V 0, D 2_{1}\right)$.

a2 is the second answer, $\mathrm{V} 0=\mathrm{a} \wedge \mathrm{V} 1>75 \wedge \mathrm{V} 1<85$. It is neither more particular nor more general than $a 1$.

s7 is obtained from the state $\mathrm{s} 3$ by consuming a 2 .

s8 is a final state.

$\mathbf{a 3}$ is the third answer, $\mathrm{VO}=\mathrm{b} \wedge \mathrm{V} 1>125 \wedge \mathrm{V} 1<135$. It is neither more particular nor more general than $a 1$ or a2.

s9 is obtained from the state 33 by consuming, a3.

s10 is a failed derivation because the resulting constraint store is inconsistent, $\mathrm{V} 1<150 \wedge \ldots \wedge \mathrm{V} 1>125+\mathrm{D} 2_{1} \wedge \mathrm{D} 2_{1}>25$. Its child is a fail node.

Note that CLP execution entered a loop resolving the state s3. Under TCLP, answer resolution avoids looping and the resulting TCLP forest is finite and complete (i.e., the leaves of the trees are either fail nodes or answers).

\section{A.4 TCLP Forest of dist/3 with Right Recursion}

Fig. A 4 shows the TCLP forest corresponding to querying the right recursive dist/3 program (Fig. ??, right). This example is useful to show how the algorithm works with mutually dependent generators ${ }^{2}$ and to see why not all the answers from a generator may be directly used by its consumers.

Unlike the left-recursive version, which shows only one TCLP tree (Fig. A 3, bottom), Fig. A 4 has two TCLP trees (one for each generator). That is because the left recursive version only sought paths from the node a, but the right recursive version creates a new TCLP tree at the state $s 4$ to collect the paths from the node $b$, since edge $(a, b)$ had been previously evaluated at state 33 . As before we only explain how we obtain some of the states:

s1 the TCLP tree $\tau_{P}(\operatorname{dist}(\mathrm{a}, \mathrm{VO}, \mathrm{V} 1), \mathrm{V} 1<150)$ is created.

s4 is obtained by resolving the literal edge $\left(\mathrm{a}, \mathrm{Z}_{1}, \mathrm{D} 1_{1}\right)$.

Ans(s5) the tabled literal dist (b,VO,D2 $2_{1}$ is a new generator and a new TCLP tree $\tau_{P}(\operatorname{dist}(\mathrm{b}, \mathrm{V} 2, \mathrm{~V} 3), \mathrm{V} 3>0 \wedge \mathrm{V} 3<100)$ is created (Def. ??).

s5 is the root node of the new TCLP tree.

s6i/ii are obtained by resolving the literal dist (b, V2, V3) against the clauses of the program.

s8 is obtained by resolving the literal edge $\left(b, Z_{1}, D 1_{1}\right)$. In the state $s 8$, the call $\left\langle\operatorname{dist}\left(\mathrm{a}, \mathrm{V} 2, \mathrm{D} 2_{1}\right), \mathrm{D} 2_{1}>0 \wedge \mathrm{D} 2_{1}<75\right\rangle$ is suspended because it entails the former generator $\left\langle\operatorname{dist}\left(\mathrm{a}, \mathrm{VO}_{1}, \mathrm{~V} 1_{1}\right), \mathrm{V} 1_{1}<150\right\rangle$.

\footnotetext{
${ }^{2}$ I.e., generators which consume answers from each other.
} 


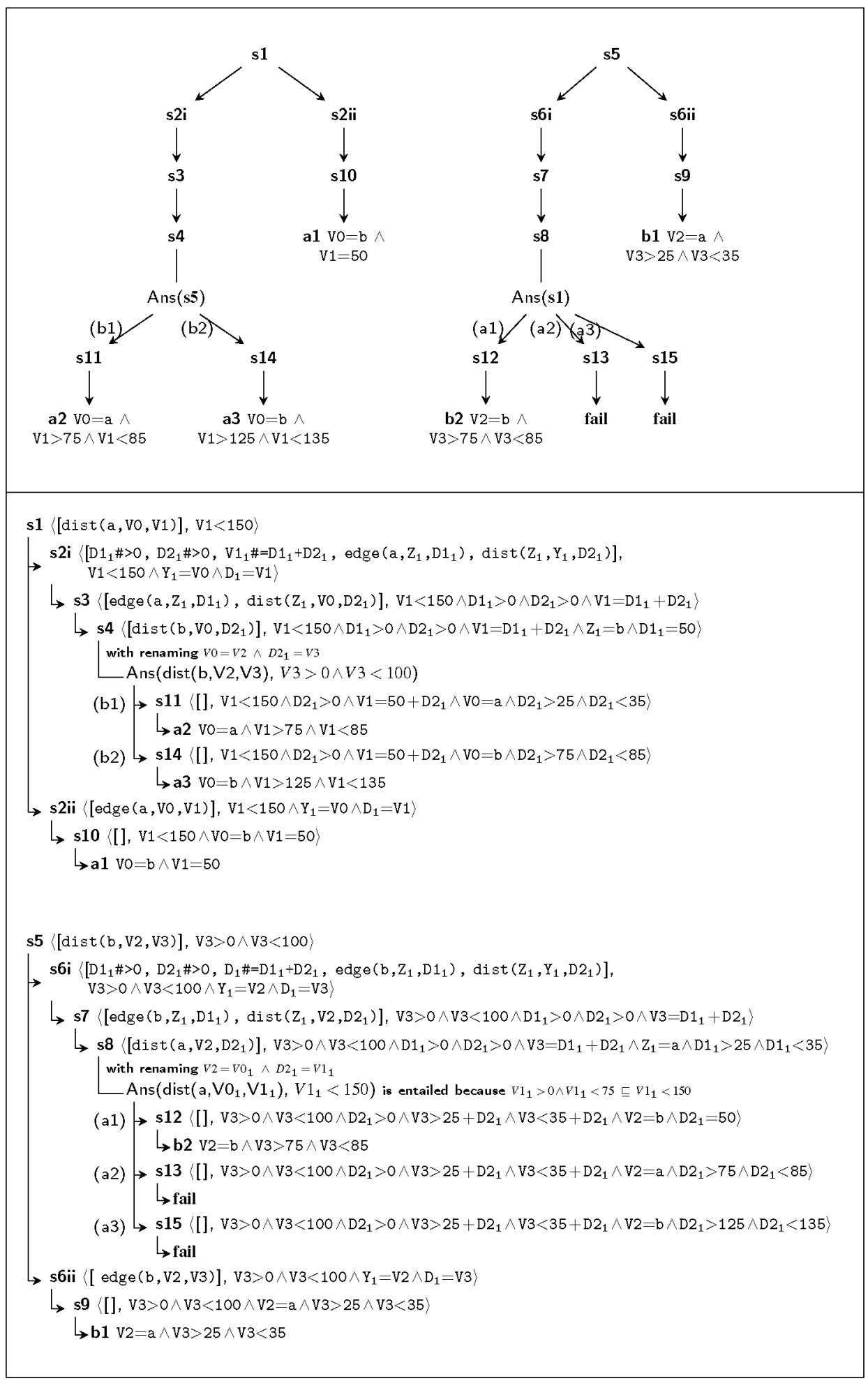

Fig. A 4: TCLP-forest of the query ?- D \#<150, $\operatorname{dist~(a,Y,D)~}$ for dist $/ 3$ with right recursion 
Ans(s1) the tabled literal $\operatorname{dist}\left(\mathrm{a}, \mathrm{V} 2, \mathrm{D} 2_{1}\right)$ is resolved with answer resolution (Def. ??) using the answers from the previous TCLP tree $\tau_{P}\left(\operatorname{dist}\left(\mathrm{a}, \mathrm{VO} \mathrm{O}_{1}, \mathrm{~V} 1_{1}\right), \mathrm{V} 1_{1}<150\right)$ because the renamed projection ${ }^{3}$ of the current constraint store onto the variable of the literal entails the projected constraint store of the generator: $\left(\mathrm{V} 1_{1}>0 \wedge \mathrm{V} 1_{1}<75\right) \sqsubseteq V 1_{1}<150$. Since the initial TCLP forest is under construction and depends on itself, the current branch derivation is suspended.

This suspension also causes the former generator to suspend at the state s4.

s9 is a final state obtained upon backtracking to the state s6ii.

b1 is the first answer of the second generator.

At this point the suspended calls can be resumed by consuming the answer b1 or by evaluating s2ii. The algorithm first tries to evaluate s2ii and then it will resume s4 consuming b1.

s10 is a final state obtained upon backtracking to the state $\mathrm{s} 2 \mathrm{ii}$.

a1 is the first answer of the first generator: $\mathrm{V0}=\mathrm{b} \wedge \mathrm{V} 1=50$.

s11 is a final state obtained from the state $s 4$ by consuming $b 1$.

$\mathbf{a} 2$ is the second answer of the first generator: $\mathrm{VO}=\mathrm{a} \wedge \mathrm{V} 1>75 \wedge \mathrm{V} 1<85$.

$\mathbf{s 1 2}$ is a final state obtained from the state s8 by consuming a1.

b2 is the second answer of the second generator.

$\mathbf{s 1 3}$ is a failed derivation obtained from s8 by consuming a2. It fails because the constraints $\mathrm{VO}=\mathbf{a} \wedge \mathrm{V} 1>75 \wedge \mathrm{V} 1<85$ are inconsistent with the current constraint store. Note that the projection of the constraint store of $s 8$ onto $V 1$ is $V 1>0 \wedge$ $\mathrm{V} 1<75$. Its child is a fail node.

s14 is a final state obtained from the state s4 by consuming $b 2$.

$\mathbf{a 3}$ is the third answer of the first generator: $\mathrm{VO}=\mathrm{b} \wedge \mathrm{V} 1>125 \wedge \mathrm{V} 1<135$.

$\mathbf{s 1 5}$ is a failed derivation obtained from s 8 by consuming a3. Its child is a fail node.

This example illustrates why left recursion reduces the execution time and memory requirements when using tabling / TCLP: left recursion will usually create fewer generators. We have also seen that using answers from a more general call, as in the answer resolution of state s8 (i.e., the constraint store of the consumer $\mathrm{V} 1_{1}>0 \wedge \mathrm{V} 1_{1}<75$ is more particular than the constraint store of the generator $\mathrm{V} 1_{1}<150$ ), makes it necessary to filter the correct ones (i.e., answer resolution for a 2 and a 3 failed). This is not required in variant tabling because the answers from a generator are always valid for its consumers (modulo variable renaming).

\section{Appendix B Step by Step Execution of dist/3 under TCLP(Q)}

The trace below shows the step by step execution of the TCLP version of the left recursive distance traversal program in Fig. ??, left, with the query ?- D \#<150, $\operatorname{dist~}(\mathrm{a}, \mathrm{Y}, \mathrm{D})$. using the graph in Fig. A 1 . In this example we are using the TCLP(Q) interface (Section ??). Each step is annotated with the labels used in Fig. ??. The execution starts with the query ?- D \#< 150, dist (a, Y,D):

\footnotetext{
3 The projection of $\mathrm{V} 3>0 \wedge \mathrm{V} 3<100 \wedge \mathrm{D} 1_{1}>0 \wedge \mathrm{D} 2_{1}>0 \wedge \mathrm{V} 3=\mathrm{D} 1_{1}+\mathrm{D} 2_{1} \wedge \mathrm{Z}_{1}=\mathrm{a} \wedge \mathrm{D} 1_{1}>25 \wedge \mathrm{D} 1_{1}<35$ onto $\mathrm{D} 2_{1}$ is $\mathrm{D} 2_{1}>0 \wedge \mathrm{D} 2_{1}<75$. After renaming $\mathrm{D} 2_{1}=\mathrm{V} 1_{1}$, the resulting projection is $\mathrm{V} 1_{1}>0 \wedge \mathrm{V} 1_{1}<75$.
} 
0 the constraint $\mathrm{D} \#<150$ in the query is added to the current store (state $s 0$ ). Then $\langle\operatorname{dist}(a, Y, D), D<150\rangle$ is called and the tabling engine takes the control of the execution calling tabled_call (dist_aux ( $a, Y, D)$ ).

1 call_lookup_table/3 initializes and saves (after renaming) dist_aux (a,Vo,V1), because it is the first occurrence, and returns Vars $=[D]$ and Gen $=\$ 1$, where $\$ 1$ is the reference for this generator.

2 store_projection([D], ProjStore) returns ProjStore=([V1], [V1\#<150]).

3 member $/ 2$ fails because the list of projected constraint stores associated to Gen $=\$ 1$ is empty.

4 save_generator/3 saves ( [V1] , [V1\#<150]) in the list of projected constraint stores associated to Gen $=\$ 1$ (state $\mathrm{s} 1$ ).

7 execute_generator/ 2 evaluates the generator against the first clause of dist_aux $/ 3$ and adds the body of the clause to the resolvent of the state $s 2 i$. Then the constraints of the resolvent, $[D 1 \#>0, D 2 \#>0, D \#=D 1+D 2]$, are added to the constraint store (state s3) and $(\operatorname{dist}(\mathrm{a}, \mathrm{Z}, \mathrm{D} 1), \mathrm{D}<150 \wedge \mathrm{D} 1>0 \wedge \mathrm{D} 2>0 \wedge \mathrm{D}=\mathrm{D} 1+\mathrm{D} 2\rangle$ is called.

0 the tabling engine reenters the tabled execution with tabled_call (dist_aux (a,Z,D1)).

1 call_lookup_table(dist_aux (a,Z,D1), Vars,Gen) returns Vars=[D1] and $\mathrm{Gen}=\$ 1$, the reference to the previous generator, dist_aux (a, V0, V1).

2 store_projection([D1], ProjStore) returns ProjStore=([V1], [V1\#>0, $\mathrm{V} 1 \#<150]$ ). For clarification, the projection of the current constraint store $\mathrm{D}<150 \wedge$ $\mathrm{D} 1>0 \wedge \mathrm{D} 2>0 \wedge \mathrm{D}=\mathrm{D} 1+\mathrm{D} 2$ onto $\mathrm{D} 1$ is $\mathrm{D} 1>0 \wedge \mathrm{D} 1<150$.

3 member $/ 2$ retrieves the projected constraint store ProjStore_G $=([V 1]$, $[\mathrm{V} 1 \#<150])$.

5 call_entail $/ 2$ succeeds because $(D<150 \wedge D 1>0 \wedge D 2>0 \wedge$ $\mathrm{D}=\mathrm{D} 1+\mathrm{D} 2) \sqsubseteq \mathrm{D} 1<150$.

6 suspend_consumer/1 suspends the current call dist_aux (a, Z,D1) (state s3, waiting for the answer of the current TCLP tree, Ans (s1)).

7 The evaluation of the generator backtracks to evaluate the other clause (state s2ii). Now the current constraint store is $\mathrm{D} \#<150$ and the call (edge $(\mathrm{a}, \mathrm{Y}, \mathrm{D}), \mathrm{D}<150$ ) unifies with edge $(a, b, 50)$ (state $s 4)$. The first answer is found and new_answer/0 is invoked to collect the answer.

8 answer_lookup_table/2 stores the Herbrand constraints ${ }^{4}$ of the answer, $\mathrm{Y}=\mathrm{b} \wedge \mathrm{D}=50$, returning $\operatorname{Var} \mathrm{s}=[]$ and $A n s=\$ \mathrm{a} 1$, where $\$ \mathrm{a} 1$ is the reference for this answer.

9 store_projection/2 returns ProjStore=([], [] ).

10 member/2 fails because the list of projected constraint stores associated to $\$ \mathrm{a} 1$ is empty.

11 save_answer/2 saves ( []$,[]$ ) in the list of the answer constraints associated to $\$ a 1$ (state a1). The first answer is collected.

14 the tabling engine resumes the goal suspended at state $s 3$ and member $/ 2$ retrieves the Herbrand constraints $Y=b \wedge D=50$ and the answer constraint $([],[]$ ).

\footnotetext{
${ }^{4}$ In solvers written in Prolog and implemented using attributed variables, such as CLP(Q) and CLP(R), it is usual that variables lose their association with the constraints where they appeared when these variables become ground. As ground terms do not have attributes attached, $D=50$ is handled as part of the Herbrand constraints.
} 

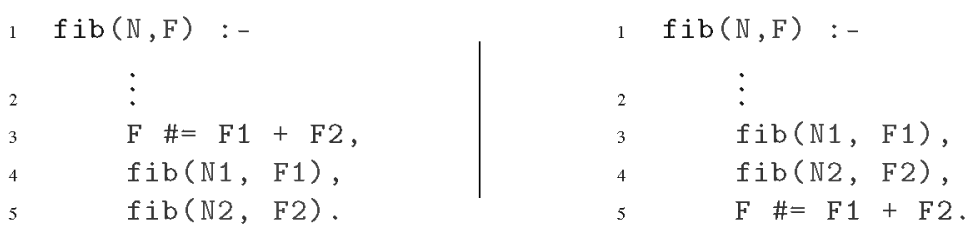

Fig. C 1: Two versions of $\mathrm{fib} / 2: \mathbb{Q}$ and $\mathbb{R}$ (left) vs. $\mathbb{D}_{\leq}$(right).

15 apply_answer/ 2 adds the answer to the current constraint store (state s5).

7 the execution continues resolving $\langle$ edge $(b, Y, D 2), \ldots \wedge D 2<100\rangle$ which unifies with the clause edge (b,a, D2) : - D2\# $>25, \mathrm{D} 2 \#<35$ (state 56 ). The second answer is found.

8 answer_lookup_table/2 stores the Herbrand constraints of the answer, vo =a, returning Vars $=[D]$ and Ans $=\$ a 2$.

9 store_projection/2 returns ProjStore=( [V1], [V1\#>75,V1\#<85]).

10 member $/ 2$ fails because the list of projected constraint stores associated to Ans $=\$ a 2$ is empty.

11 save_answer/2 saves ( $[\mathrm{V} 1],[\mathrm{V} 1 \#>75, \mathrm{~V} 1 \#<85]$ ) in the list of answer constraints associated to $\$ \mathrm{a} 2$ (state a2). The second answer is collected.

14, 15, 7 the tabling engine resumes the suspended goal at state $s 3$ and consumes the second answer following the same steps as with the first one and generating the states $\mathrm{s} 7$ and s8. The third answer has been found.

$\mathbf{8 , 9}, 10,11$ the answer is collected and ( $[\mathrm{V} 1],[\mathrm{V} 1 \#>125, \mathrm{~V} 1 \#<135])$ is saved in the list of answer constraint associated to \$a3 (state a3).

14, 15 the tabling engine resumes the suspended goal at state $s 3$ and consumes the third answer.

7 the execution fails resolving $\left\langle\right.$ edge $\left.\left(b, V 0, D 2_{1}\right), V 1<150 \wedge \ldots \wedge \mathrm{D} 1_{1}<135\right\rangle$ (states s9 and s10)

14, 15 the generator has exhausted all the answers and it does not have any more dependencies, so complete/ 0 marks the generator as complete. The query retrieves the answers from the generator one by one and returns them.

\section{Appendix C Comparison of Mod TCLP using $R, Q$ and $D_{\leq}$}

This section highlights that the modularity of TCLP makes it possible to choose the most adequate constraint solver for the specific problem, and that decision should not always be based solely on the performance of the constraint solver, but also on its expressiveness and / or precision. Since TCLP, unlike CLP, uses entailment checking extensively to decide whether to suspend and save / discard answers or not, the performance of entailment is more relevant than in CLP. It also makes its soundness (which can be challenged by e.g. numerical accuracy) critical, as incorrect entailment results can lead to non-termination or to unexpected termination.

We use the doubly recursive Fibonacci program (Fig. C 1). It is well-known that tabling reduces its complexity from exponential to linear, but, in addition, CLP makes it possible to run exactly the same program $\mathrm{fib} / 2$ backwards to find the index of some Fibonacci 


\begin{tabular}{|l|r|r|r|}
\hline & Mod TCLP(R) & Mod TCLP(Q) & Mod TCLP(D $\leq)$ \\
\hline fib(N, 832040) & $\mathbf{2 5}$ & 61 & 147 \\
\hline fib(N, 28657) & $\mathbf{1 6}$ & 40 & 69 \\
\hline fib(N, 610) & $\mathbf{8}$ & 19 & 24 \\
\hline fib(N, 89) & $\mathbf{5}$ & 12 & 13 \\
\hline
\end{tabular}

Table C 1: Run time (ms) comparison for the $\mathrm{fib} / 2$ using $\mathrm{R}, \mathbb{Q}$ and $\mathbb{D}_{\leq}$.

number by generating a system of equations whose solution is the index of the given Fibonacci number. Under CLP, the size of this system of equations grows exponentially with the index of the Fibonacci number. However, under TCLP, entailment makes redundant equations not to be added and solving them becomes less expensive.

We have run this benchmark using $\mathbb{R}, Q$ and $D_{\leq}$. Due to the characteristics of $\mathbb{D}_{\leq}(\mathrm{Sec}-$ tion ??), the program for this constraint system is slightly different from the ones for $Q$ and $\mathbb{R}$ (Section ??). In these two, constraints are placed before the recursive calls. However, $\mathrm{D}_{\leq}$can have at most two variables per constraint, and therefore we had to move the constraint $F \#=F 1+F 2$ to the end of the clause (Fig. C 1). This can be detrimental to the performance of Mod TCLP( $\left(\mathbb{D}_{\leq}\right)$, as value propagation in the constraints is less effective.

Table $\mathrm{C} 1$ shows the experimental results. First, note that the Mod TCLP( $\left(\mathbb{D}_{\leq}\right)$version is slower than any of the other two. While the implementation of CLP(D $\left.\mathbb{D}_{\leq}\right)$is comparatively faster than $\operatorname{CLP}(\mathbb{R})$ and $\operatorname{CLP}(\mathbb{Q})$, moving the $F \quad \#=F 1+F 2$ to the end of the clause (which is necessary to satisfy the instantiation requirements of $D_{\leq}$) reduces its usefulness to prune the generation of redundant constraints.

Additionally, note that although the solvers for $\mathbb{R}$ and $\mathbb{Q}$ are practically the same, $\operatorname{Mod}$ TCLP $(\mathbb{R})$ is fastest in all cases, since it uses directly CPU floating point numbers while CLP(Q) implements rational numbers by software. However, there is a drawback: floating point arithmetic is not accurate, and when CLP(R) approximates its results, it can cause (depending on the particular program) non-termination. That would be the case for a query such as ?- $\mathrm{fib}(\mathrm{N}, 23416728348467685)$, which terminates correctly with Mod TCLP(Q), but it does not (in under five minutes) with Mod TCLP(R), since the termination condition never holds. 